This item was submitted to Loughborough's Research Repository by the author.

Items in Figshare are protected by copyright, with all rights reserved, unless otherwise indicated.

\title{
Numerical study of vented hydrogen explosions in a small scale obstructed chamber
}

PLEASE CITE THE PUBLISHED VERSION

https://doi.org/10.1016/j.ijhydene.2018.07.078

PUBLISHER

Elsevier ( Hydrogen Energy Publications

VERSION

AM (Accepted Manuscript)

\section{PUBLISHER STATEMENT}

This paper was accepted for publication in the journal International Journal of Hydrogen Energy and the definitive published version is available at https://doi.org/10.1016/j.ijhydene.2018.07.078

\section{LICENCE}

CC BY-NC-ND 4.0

\section{REPOSITORY RECORD}

Li, Ruipengyu, Weeratunge Malalasekera, and Salah Ibrahim. 2018. "Numerical Study of Vented Hydrogen Explosions in a Small Scale Obstructed Chamber". Loughborough University.

https://hdl.handle.net/2134/34073. 


\title{
Numerical study of vented hydrogen explosions in a small scale obstructed chamber
}

\author{
Ruipengyu Li $^{\mathrm{a}, *}$, Weeratunge Malalasekera ${ }^{\mathrm{a}}$, Salah Ibrahim ${ }^{\mathrm{b}}$ \\ ${ }^{a}$ Wolfson School of Mechanical, Electrical and Manufacturing Engineering, Loughborough \\ University, Loughborough, LE11 3TU, UK \\ ${ }^{b}$ Department of Aeronautical and Automotive Engineering, Loughborough University, \\ Loughborough, LE11 3TU, UK
}

\begin{abstract}
There is a growing need to understand and estimate the explosion hazards associated with hydrogen storage and utilisation. This paper presents a comprehensive numerical study on the explosion characteristics of a lean hydrogen-air mixture in a small-scale obstructed vented chamber. The large eddy simulation (LES) technique is employed to study the highly unsteady turbulence-driven explosion when the flame propagates past successive obstructions. A dynamic flame surface density (DFSD) model is applied to the filtered chemical source term in the LES to account for the progressive wrinkling of the deflagrating flame. The driving mechanism of pressure rise and the underlying physics of flame-obstacle interactions are illustrated using the detailed LES results. The paper considers 11 individual flow experimental configurations of various obstacle number, size and location. They are further classified into six groups to investigate the influence of the level of blockage and the separation distance between adjacent obstructions. Critical safety-related parameters including the maximum overpressure and its incidence time are analysed. A comparison with propane is also made to highlight the substantial overpressure and flame acceleration of hydrogen deflagrations. Satisfactory agreements have been obtained between the LES and the experimental data, and this confirms the capability of the developed computational models in capturing essential explosion features and information for the study of vented hydrogen explosions.
\end{abstract}

Keywords: Hydrogen explosions, Flame-obstacle interaction, Dynamic LES model, Overpressure, Flame wrinkling

\footnotetext{
*Corresponding author

Email address: r.li3@lboro.ac.uk (Ruipengyu Li)
} 


\section{Introduction}

Hydrogen $\left(\mathrm{H}_{2}\right)$ as an alternative fuel and an energy carrier has many benefits due to its high heat value, renewable capability and the exclusion of harmful emissions [1]. The utilisation of hydrogen lies in hydrogen fuel cell electric vehicles [2, 3], hydrogen-fuelled internal combustion engines [4] and heating in buildings and industry [5]. However, some of its properties require additional engineering controls and considerations to ensure the safe use $[6,7]$. As hydrogen is much lighter than air and rapidly dissipates when released, a leak often leads to fast mixing with surrounding air. Also, it has a wide range of flammability limits and relatively low ignition energy, making burning and accidental explosion of hydrogen-air mixtures more likely. The situation may be less severe in an open space since $\mathrm{H}_{2}$ rises quickly into the atmosphere, but it can be a dangerous gas in confined or partially-confined regions involved in its production, storage, transport and end-user application [8].

Explosion hazards and safety issues while working with hydrogen has been a significant concern in its related storage, buildings and processing plants [7-10]. Compared with other common fuels such as methane $\left(\mathrm{CH}_{4}\right)$ and propane $\left(\mathrm{C}_{3} \mathrm{H}_{8}\right)$, hydrogen explosions are potentially more dangerous due to its high combustion speed and excessively generated overpressure. The possibility of hydrogen leaks and subsequent explosions in situations such as tunnels and refuelling stations $[7,11]$ necessitates an improved understanding of the features and behaviours of hydrogen explosions. Yanez et al. [12] reported the production, discharge, accumulation and explosion of hydrogen during the Fukushima-Daiichi accident (March 11th 2011) and highlighted the devastating consequence even with the amount of $\mathrm{H}_{2}$ involved.

Methods for protecting structures from internal explosions including venting and suppression require the knowledge of the explosion characteristics $[13,14]$. It is known that the generated overpressure significantly contributes to the destruction of an explosion event. The overpressure is dependent on various conditions such as the fuel/air equivalence ratio and ignition location. Furthermore, the surrounding obstacle-generated turbulence can significantly enhance the explosion overpressure. 
Thus an appropriate estimation of hydrogen explosion hazards has to consider the effect of obstacles and turbulence. There has been a significant amount of experimental work regarding the explosion features of $\mathrm{H}_{2}$. Besides medium or large sale explosion tests [15], a few lab-scale experimental studies [16-19] have been conducted in order to gain insights into the factors that influence the hydrogen-explosion behaviours such as vent area [18], vent burst pressure [16] and the position of ignition sources $[17,18]$.

Computational fluid dynamics (CFD) is evolving as an efficient and reliable alternative to experiments and has been used in safety-related research in buildings and off-shore processing plants [20-23]. There has also been literature related to hydrogen dispersion [7, 24-26], high-speed propagating hydrogen flames [27], impinging hydrogen jet flame [28] and hydrogen explosions [8, 29, 30]. The abundant results obtained from CFD simulations such as the overpressure and the detailed flow field are particularly useful in assisting the assessment and the design of equipment, buildings and other structures. For example, Hansen et al. [13] have recently extracted and estimated the explosion loads (actual forces) on the equipment with different geometrical shapes from CFD simulations in large-scale scenarios using the commercial software FLACS. Furthermore, performing a large number of well-validated simulations can provide a database for various operating conditions including the gas concentration, object geometries, vent area size, etc., and such statistically analysed data can help to propose new correlations as a fast and efficient tool for engineers to estimate overpressure in gas explosions [31]. It is hoped that CFD results will potentially help to give guidance to the engineers and safety consultants, e.g. determining the optimal separation distance between adjacent buildings in a congested area and the design strength of facilities. Despite the beneficial information provided for hazards and risk analysis of hydrogen technologies, applying CFD models should be with cautiousness regarding their limitations and accuracy in certain cases. Vyazmina and Jallais [29] pointed out that an explosion model validated for large-scale enclosures may not be well-adapted for small-scale chambers $\left(<1 \mathrm{~m}^{3}\right)$. 
Eventually, new CFD models for safety issues should go through a series of rigorous steps of validation and evaluation before being available to the end users, and this is also the idea of the model evaluation protocol for CFD assessment of hydrogen safety issues [32], recently proposed in the hydrogen community.

Although considered cost-effective in exploring explosion hazards and designing safer structures, the accuracy of the CFD method still relies on the modelling approaches for turbulence and combustion [29, 33]. For large-scale industrial applications, the use of Reynolds-averaged Navier-Stokes (RANS) [11, 29, 30, 32-35] has been dominant due to the computational efficiency and moderate accuracy. Within the academic research on gas explosions, the large eddy simulation (LES) based models are becoming more popular [36-42]. Gubba et al. [40] and Di Sarli et al. [41] have demonstrated that LES can be used to study and understand the sophisticated features of vented explosions in the presence of obstacles. Also, Molkov et al. [39] applied and validated an LES combustion model for hydrogen/air deflagration in a large-scale (78.5 m long) obstructed tunnel. The significant advantages of LES such as the ability to sufficiently account for the primary features of turbulent flows, as well as resolving the highly unsteady flame propagation may compensate the extra computational cost compared with RANS. For example, Di Sarli et al. [41] obtained an improved prediction of overpressure and flame speed using LES compared to the RANS simulation performed previously by Patel et al. [43] in the same explosion chamber.

Since modelling techniques for turbulent premixed flames are used for most gas explosions, an essential factor to consider when using LES is the sub-grid scale (SGS) combustion model as no portion of the filtered chemical source term can be resolved on an LES grid [44]. Another complexity lies in if the SGS model can adequately account for the effects of obstructions that are generally present in hydrogen production and processing facilities. The wrinkling and distortion of the deflagrating flame front significantly increase as it interacts with surrounding obstacles and the explosion can start from initially laminar and progress to be fully turbulent. Among 
the available SGS models in the literature, the flame surface density (FSD) based models have been applied in a wide range of test cases of premixed combustion $[45,46]$. FSD based approaches in LES often include solving a transport equation for the reaction progress variable with the filtered source term commonly closed by using algebraic formulations [47, 48] or solving an additional transport equation [49]. The algebraic FSD approaches have been used in several explosion-related studies $[34,36,40,41]$ due to its simplicity and robustness. However, as the model parameter is typically fixed in the simulation by the user, it may fail to predict the explosion features with a wide range of obstacle configurations and operating conditions.

In this paper, the characteristics and vital safety-related parameters of vented hydrogen explosions in a small scale chamber with obstructions have been studied using a dynamic flame surface density (DFSD) combustion model. Based on the renowned Boger et al. [47] algebraic model, the DFSD model evaluates the model coefficient on-the-fly, according to the information of the resolved flame front. Wang et al. [50] first proposed the model for simulating a growing turbulent flame kernel, while our study aims to apply and assess it in a more complex environment including flame-obstacle interactions and laminar to turbulent transition. Compared with some of the earlier numerical explosion studies such as [29], the main contribution made in this study is the insight into the pressure generation mechanism, specifically in small-scale ( $<1$ litre) turbulence-driven vented hydrogen explosions with multiple obstructions, as well as an assessment of the recently developed LES-DFSD approach using a series of test configurations. The objectives of this paper are twofold: $(i)$ to study and understand the essential features of the vented hydrogen deflagrations and the influences of obstructions (e.g. number, position and size of obstacles) using LES; (ii) to evaluate the capability of the developed DFSD model in predicting the overpressure and flame acceleration in considered configurations and compared them with recent experimental data. The results presented in the paper intend to demonstrate the benefits and potential of LES as a computational tool for a range of types and scales of hydrogen explosions. 


\section{Experimental setup}

The recent experiments from the University of Sydney [51] are used to validate the developed computational model and to study the explosion characteristics of hydrogen. The explosion chamber is shown schematically in Figure 1. It has a square cross-section with a side of $50 \mathrm{~mm}$, and the length is $250 \mathrm{~mm}$, giving a volume of 0.625 litres and an aspect ratio of 5:1. The chamber can accommodate three removable baffle plates (also referred to as grids or obstacles in the rest of the paper) with a schematic shown in Figure 1b. Each baffle consists of five 4-mm wide and 3-mm thick strips evenly separated by six 5-mm gaps, producing an area blockage ratio (ABR) of $40 \%$ in the flow direction. A single baffle also creates a volume blockage ratio (VBR), i.e. the ratio of blocked volume to the total volume of the chamber, of $0.48 \%$. These may be located at any of the three locations: $19 \mathrm{~mm}$ (B1), $49 \mathrm{~mm}$ (B2) and $79 \mathrm{~mm}$ (B3) from the base. A further solid obstruction with a square cross-section can be placed such that its lower surface is $96 \mathrm{~mm}$ away from the base plate. Two solid obstacles may be used, a small one with a cross-section of $12 \times 12 \mathrm{~mm}$ or a large one with a size of $25 \times 25 \mathrm{~mm}$. They create ABRs of $24 \%$ and $50 \%$ (VBRs of $1.152 \%$ and $5 \%$ ), respectively.

While the focus of this paper is on hydrogen, the experiment also uses two other fuels, namely liquid petroleum gas (LPG) and compressed natural gas. In the experiment, the fuel-air mixture enters the atmospheric pressure chamber through a non-return valve, and it is allowed to rest before each ignition event. The stagnant fuel-air mixture is then ignited by focusing the infrared output from an Nd: YAG laser $2 \mathrm{~mm}$ above the base, and this sets the time zero for each experimental run. The hinged flap at the chamber exit rises 1 second before ignition to allow venting throughout the explosion. Pressure is recorded at $25 \mathrm{kHz}$ using two Keller type PR21-SR piezo-electric pressure transducers located in the base plate as well as in the wall of the chamber. High-speed imaging of laser-induced fluorescence from $\mathrm{OH}$ (LIF-OH) with a repetition rate of $5 \mathrm{kHz}$ is also performed providing an excellent representation of the evolution of the reaction zones. 


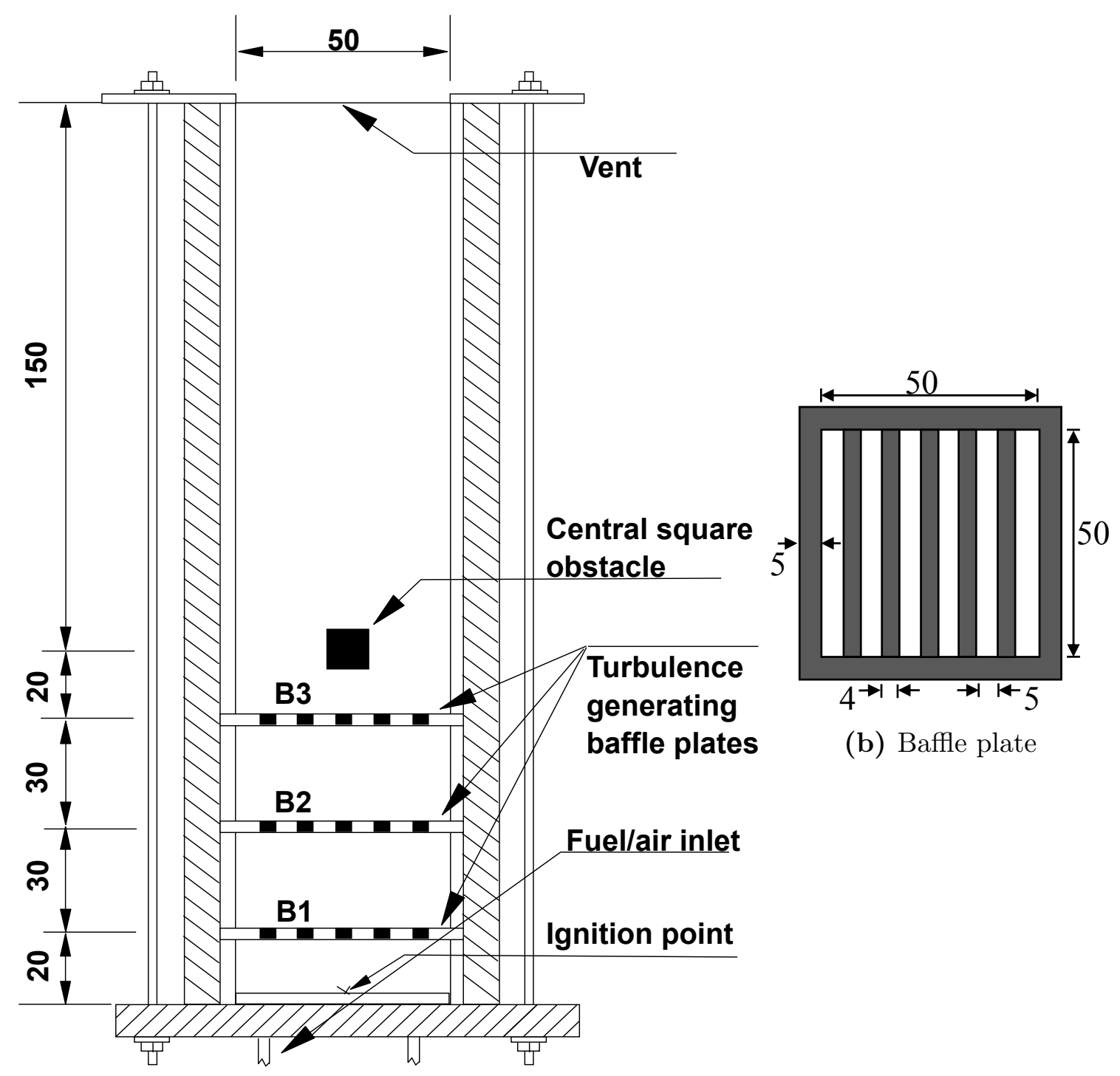

(a) Chamber

Figure 1: Specifications of the explosion chamber used in the experiment [51] (not to scale, dimensions are in $\mathrm{mm}$ ).

Table 1: Grouped configurations to study the influence of obstructions

\begin{tabular}{lll}
\hline Group & \multicolumn{1}{c}{ Description } & Configuration \\
\hline 1 & $\begin{array}{l}\text { Baffles are progressively increased and } \\
\text { kept furthest from ignition end }\end{array}$ & 00BS, 0BBS, BBBS \\
2 & $\begin{array}{l}\text { Baffles are progressively increased from } \\
\text { ignition end }\end{array}$ & B00S, BB0S, BBBS \\
3 & $\begin{array}{l}\text { Two baffles are positioned at different } \\
\text { stations of the chamber }\end{array}$ & BB0S, B0BS, BBBS \\
4 & $\begin{array}{l}\text { One baffle is positioned at a different } \\
\text { station of the chamber }\end{array}$ & B00S, 0B0S, 00BS \\
5 & $\begin{array}{l}\text { Central obstacle size increases with all } \\
\text { three baffle plates present }\end{array}$ & BBB0, BBBS, BBBL \\
6 & $\begin{array}{l}\text { Small or large central obstacle without } \\
\text { any baffle plates }\end{array}$ & 000S, 000L \\
\hline
\end{tabular}


A total number of 11 configurations (see supplementary material for a list of schematics) has been investigated numerically for the present study. A code is used to identify each configuration to specify the obstruction locations and size of the solid obstacle. For example, 0B0S stands for the case where one baffle plate is at $\mathrm{B} 2$, and the small central obstacle $(\mathrm{S})$ is placed downstream of the ignition point, and BBBL represents the configuration where three baffle plates B1, B2 and B3, as well as the large central obstacle (L), are positioned in the same order away from the chamber bottom.

Table 1 displays the configurations classified into six groups to facilitate the analysis of the impact of obstructions. Groups 1-4 aim to investigate the influence of the turbulence-generating baffle plates, while groups 5 and 6 intend to examine the impact made by changing the size of the central obstacle. This classification of flow configurations is made to represent various means of blockage including the number and location of the baffles as well as the size of the solid central obstacle.

\section{Numerical setup}

\subsection{The combustion model}

The premixed deflagration flame is governed by equations for conservation of mass, momentum and energy. A transport equation for a reaction progress variable $c$ is also included, representing the chemical state of the fuel-air mixture. It is defined such that $c=0$ where the mixture is unburned and $c=1$ where it is fully burned. The general form of the Favre-filtered transport equation for $\tilde{c}$ is

$$
\frac{\partial \bar{\rho} \widetilde{c}}{\partial t}+\frac{\partial}{\partial x_{i}}\left(\bar{\rho} \widetilde{u}_{i} \widetilde{c}\right)=-\frac{\partial}{\partial x_{i}}\left(\bar{\rho} \widetilde{u_{i} c}-\bar{\rho} \widetilde{u}_{i} \widetilde{c}\right)+\overline{\frac{\partial}{\partial x_{i}}\left(\rho D \frac{\partial \widetilde{c}}{\partial x_{i}}\right)}+\overline{\dot{\omega}}_{c}
$$

where the filtered source term, $\overline{\dot{\omega}}_{c}$, can be closed using the concept of the flame surface density as

$$
\overline{\dot{\omega}}_{c}=\rho_{u} S_{L}^{0} \Sigma
$$


where $\rho_{u}$ is the density of the fresh gases, and $S_{L}^{0}$ is the unstrained laminar burning velocity. $\Sigma$ is the sub-grid scale flame surface density, which is expressed using the algebraic formulation [45]:

$$
\Sigma=4 \sqrt{\frac{6}{\pi}} \Xi_{\Delta} \frac{\widetilde{c}(1-\widetilde{c})}{\Delta}
$$

where $\Delta$ is the combustion filter width and $\Xi_{\Delta}$ is the sub-grid scale flame wrinkling factor. Thus, the $\widetilde{c}$-equation adopted for the present study is $[45,50]$

$$
\frac{\partial \bar{\rho} \widetilde{c}}{\partial t}+\frac{\partial}{\partial x_{i}}\left(\bar{\rho} \widetilde{u}_{i} \widetilde{c}\right)=\frac{\partial}{\partial x_{i}}\left(\frac{\rho_{u} \Xi_{\Delta} S_{L}^{0} \Delta}{16 \sqrt{6 / \pi}} \frac{\partial \widetilde{c}}{\partial x_{i}}\right)+4 \rho_{u} S_{L}^{0} \sqrt{\frac{6}{\pi}} \Xi_{\Delta} \frac{\widetilde{c}(1-\widetilde{c})}{\Delta}
$$

where the modified diffusion term, the third term in Eq. (4), is included to control the filtered flame thickness and to reproduce the correct laminar propagation speed in the absence of sub-grid scale turbulence effect [45]. Note that $\Delta$ should be larger than the mesh size $\Delta_{x}$ (typically $\Delta \geq 5 \Delta_{x}$ ) so that the filtered progress variable gradients can be well resolved on an LES grid [50]. In the present study, $\Delta=6 \Delta_{x}$ is used.

The SGS wrinkling factor $\Xi_{\Delta}$ measures the ratio between total and resolved flame surfaces locally, and it accounts for the sub-grid scale flame surface lost by the filtering process [50]. A fractal-like expression for $\Xi_{\Delta}$, originally proposed by Wang et al. [50], is adapted for the present investigation:

$$
\Xi_{\Delta}=\left(\frac{\Delta}{\delta_{c}}\right)^{\beta}
$$

where the inner cut-off scale, $\delta_{c}$, is lost in the filtering process and it requires to be prescribed by the user. Note that $\delta_{c}$ should be of the order of the laminar flame thermal thickness, $\delta_{L}^{0}[52]$, and $\delta_{c}=4 \delta_{L}^{0}$ is used in this work. The model parameter $\beta$ is determined dynamically by comparing the test-filtered and resolved flame fronts:

$$
\beta \approx \frac{\ln (\langle|\widehat{\nabla \widetilde{c}}|\rangle /\langle|\nabla \widehat{\widetilde{c}}|\rangle)}{\ln \left(\sqrt{1+(\widehat{\Delta} / \Delta)^{2}}\right)}
$$


with $\widehat{\Delta}$ being the width of the test filter. The test-filtering ( $\widehat{\ldots}$ operator) and the spatial-averaging $(\langle\ldots\rangle$ operator $)$ procedures in Eq. (6) are carried out using an implemented Gaussian filter [53]. The test-filter and averaging-filter widths are set as $\widehat{\Delta}=1.1 \Delta$ and $\Delta_{m}=3.0 \widehat{\Delta}$, respectively. Note that $\widehat{\Delta}$ should be larger than the filtered flame thickness (typically, $\widehat{\Delta} \geq \Delta$ ) [52], and the size of $\Delta_{m}$ has been found to have a minimal effect on the results presented in this paper. The laminar burning velocity and thermal flame thickness are set as $S_{L}^{0}=125 \mathrm{~cm} / \mathrm{s}[54]$ and $\delta_{L}^{0}=0.12 \mathrm{~mm}$ [55], respectively, for the lean hydrogen-air mixture of equivalence ratio $\Phi=0.7$. In the simulation of the deflagration, Eqs. (5) and (6) are used to automatically adjust the degree of SGS flame wrinkling $\Xi_{\Delta}$ based on the instantaneous resolved flame characteristics.

\subsection{Computational details}

The LES computation of the vented hydrogen deflagration has been carried out using the in-house code PUFFIN [56]. The code solves the compressible form of mass, momentum and scalar equations, discretised using a finite volume formulation on a Cartesian grid. The Gaussian spatial filtering and the DFSD model using Eqs. (4) to (6) have been implemented in the code to account for the potential progressive flame wrinkling in the deflagration.

The domain of the explosion chamber has dimensions of $50 \times 50 \times 250 \mathrm{~mm}$, and the size of the whole computational domain is $325 \times 325 \times 500 \mathrm{~mm}$. A non-reflecting boundary condition [46] is applied to the boundaries of the far field, to minimise the effect of reflected pressure waves on the internal pressure field of the chamber. The chamber domain constitutes $85 \times 85 \times 423$ cells in the $x, y$ and $z$ directions, respectively. They are distributed uniformly in the chamber giving a grid size of $\Delta_{x} \approx 0.59$ mm. The mesh size is chosen to ensure that the filtered flame thickness $(\approx \Delta)$ is smaller than the gaps between the strips of the baffle plates. The grid is expanded from the chamber toward the far-field boundaries to save the computational time. Adiabatic and no-slip boundary conditions are employed on the solid walls of the chamber, and the 1/7th power law wall function of Werner and Wengle [57] is used 
to calculate the shear stress at the solid wall. Subgrid-scale turbulence is modelled using the dynamic Smagorinsky eddy viscosity model [58].

Conservation equations for scalars use second-order central difference scheme for diffusion terms. SHARP [59] is used for advection terms of the scalar equations to avoid problems associated with oscillations in the solution. Time integration of scalar equations uses the Crank-Nicolson scheme. Further details of the numerical scheme are given elsewhere [see 56] and are thus not presented here. The initial velocity field is quiescent, with a random perturbation to generate initial turbulence. Flame is initialised using a burned flame kernel pre-filtered at the filter width $\Delta$. It is superimposed at the ignition point of the domain to initiate each simulation. The kernel is hemispherical and has a radius of $3 \mathrm{~mm}$ for all the simulations. Two other computational grids $\left(\Delta_{x} \approx 1 \mathrm{~mm}\right.$ and $\Delta_{x} \approx 0.75 \mathrm{~mm}$ within the chamber $)$ have been investigated to examine the solution dependence on the mesh resolution, and the variation in the maximum overpressure is found to be small $(<4 \%)$ between the intermediate and fine (used in this paper) grids. The quality of LES for the present grid resolution ( $\Delta_{x} \approx 0.59 \mathrm{~mm}$ within the chamber) is characterised according to Popes criterion [60]. At least $80 \%$ of the total turbulent kinetic energy is resolved in the majority of the flame region, considering the fully turbulent phase of the explosion in the configuration BBBS. All the LES simulations are performed on an HP Z840 workstation with an Intel Xeon $3.5 \mathrm{GHz}$ processor. Under the current mesh resolution, a run for the case BBBS requires 312 CPU hours until the leading edge of the flame exits the explosion chamber.

\section{Results and discussion}

Numerical simulations of vented hydrogen explosions have been performed for 11 individual configurations grouped in Table 1. Section 4.1 presents a global study of the deflagration emphasising the underlying physics of the flame-obstacle interaction and the mechanism of pressure build-up within the explosion chamber. Some critical safety-related parameters including the maximum overpressure and the flame 
propagation speeds obtained from the LES are compared against the experimental results in section 4.2. The focus is to analyse the effects of obstructions such as the number and the location of the baffle plates and the size of the central obstacle. The ability of the developed computational setup in capturing the essential explosion characteristics is also assessed. Finally, the results between hydrogen and propane are discussed in section 4.3 to highlight the differences regarding their explosion characteristics.

\subsection{A phenomenological study}

The physical phenomena and the typical flame behaviours behind the hydrogenair deflagration are discussed in this section using the configuration B0BS as an example. Figure 2 shows the shape of the typical hydrogen-air explosion flame extracted experimentally and numerically for configuration B0BS. The reaction zone is marked using a sequence of high-speed images of LIF-OH in the experiment [51], which is indicated using the contour of $\widetilde{c}$ numerically from LES. Evidently from Figure 2, LES can reproduce both the flame structure and the global propagation speed. It can be noticed that the flame shape continuously changes during the explosion. Initially, the flame is laminar and expands hemispherically before impinging on the first baffle plate. It penetrates the grid and separates into four fingers primarily resulting from the 'geometric' stretch caused by the strips of the baffle plate. Then, they quickly merged with each other due to the lateral spread of the flame. Meanwhile, the surface area of the flame tends to decrease before reaching the next grid. The flame then jets through the gaps of B3 and immediately strikes the square obstacle. It can be seen that the flame front is very much corrugated when interacting with the obstacle. After passing the last obstruction (square obstacle), the flame is significantly wrinkled ( $\mathrm{t}=4.4 \mathrm{~ms}$ of the LIF-OH images) and propagates towards the chamber outlet. The blockage introduced by the central obstruction also leaves a small amount of unburned mixture behind it when wrapped by the flame front. It is then consumed when the leading flame point is further downstream, as indicated by both high-speed and LES images. It can be noticed that the flame spreads faster 


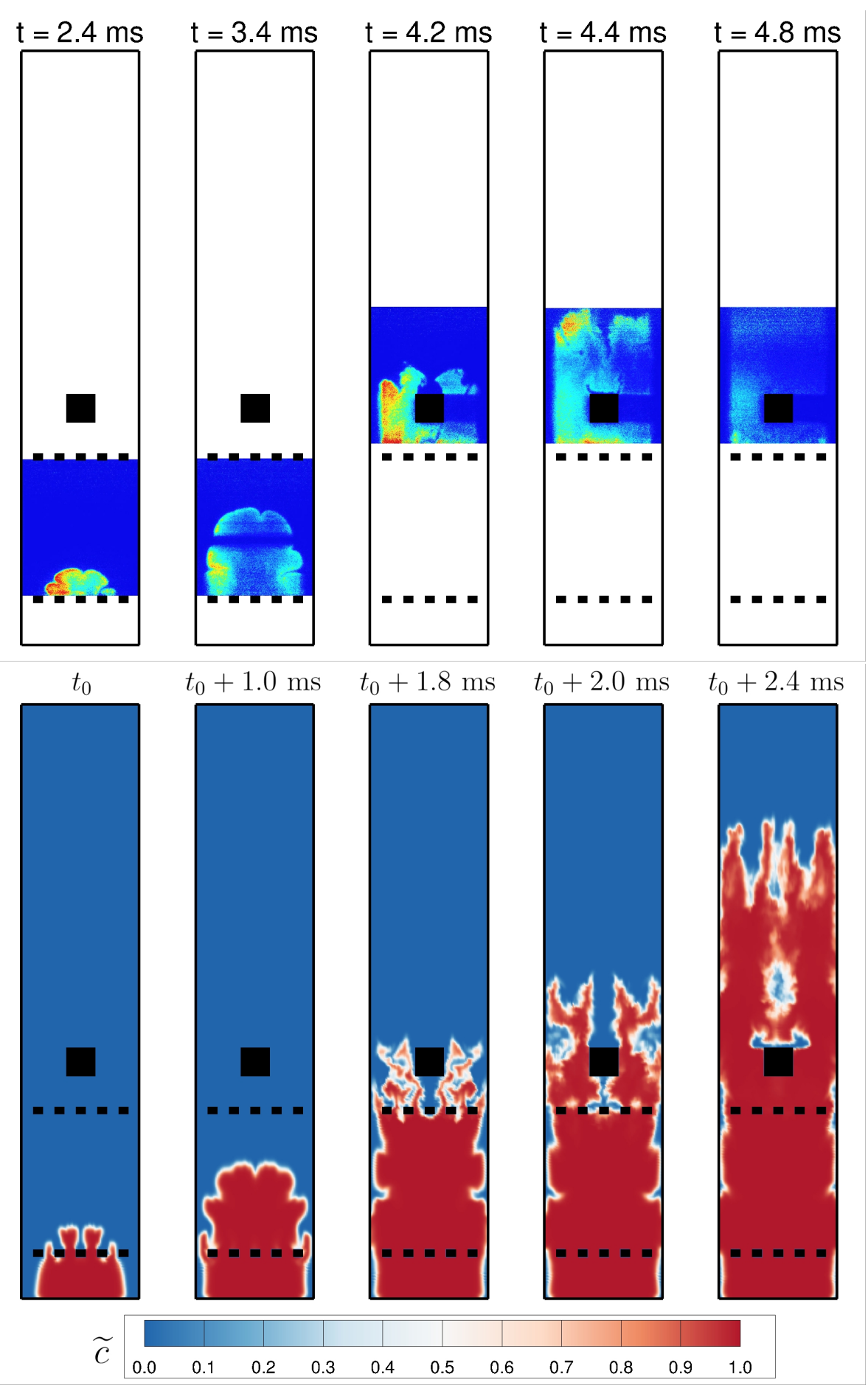

Figure 2: Comparison of deflagration flame propagation between simulations and experiments for configuration B0BS. Top: LIF-OH high-speed images from the experiments [61]. Bottom: LES flame images contoured by $\widetilde{c}$. $t_{0}$ is set to match the position of the flame leading point with the high-speed image. Time intervals between successive LES images are kept the same as the experiment. 
in the upper part of the combustion chamber due to the increased flame distortion caused by the interaction with the upstream obstacles.

Figure 3a shows the calculated overpressure distribution along the axial direction on the mid-plane of the chamber for the configuration B0BS. The pressure has been found to be nearly uniform on the $x y$-plane (i.e. cross-section) of the chamber. During the explosion process, the magnitude of the internal pressure rises as well as its spatial range of influence, which is approximately the region of the burned gases. Note that the wall pressure (downstream of the central obstacle) measured in the experiments is consistently lower than the base and it may suggest that a pressure gradient exists within the chamber [61]. It is further confirmed by LES with a negative pressure gradient vertically along the chamber, which drives the explosion gases out across the outlet. A significant pressure drop to atmospheric level due to venting can be found near the exit of the combustion chamber. Furthermore, both the numerical (not shown here) and the experimental results [61] confirm that the two pressure probes on the chamber base and the wall downstream of the central obstacle have given similar pressure patterns in all the test cases. Therefore, the analysis and discussion of the rest of this paper will focus on the pressure at the bottom of the vessel.

Figure 3b shows the overpressure history computed by LES and the corresponding gas expansion and venting strength in the explosion chamber, with the purpose of describing the trend of pressure development during the hydrogen explosion. It is known that the pressure rise in the semi-confined enclosure is a consequence of the competition between the two events: $(i)$ gas expansion caused by combustion tends to promote the pressure build-up; (ii) discharge of the explosion gases through venting tends to release the internal pressure. The volumetric flow rates of gas expansion, $\dot{V}_{\text {expa }}$ and venting, $\dot{V}_{v e n t}$ can be readily computed using the LES velocity field. $\dot{V}_{\text {expa }}$ can be represented by the rate of volume change of the fresh unburned gases with the burned gases. In LES, this may be evaluated from $\dot{V}_{\text {expa }}=\frac{\partial}{\partial t} V_{b}\left(1-\frac{\rho_{b}}{\rho_{u}}\right)$, where $\rho_{u}$ and $\rho_{b}$ are the densities of unburned and burned mixtures, respectively. The volume 


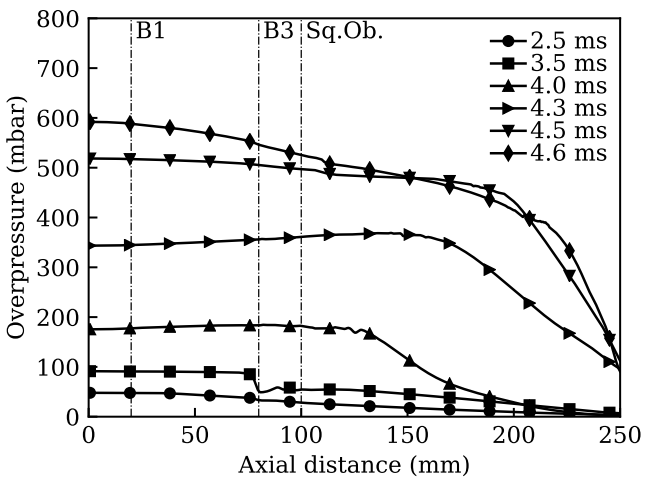

(a) Overpressure distribution along the axial direction on the mid-plane of the chamber $(\mathrm{y}=14 \mathrm{~mm}$ from the centre)

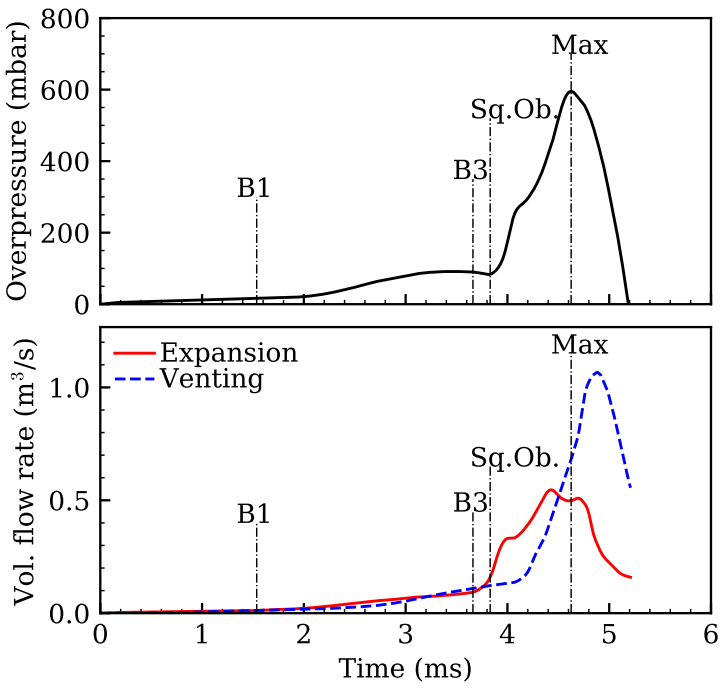

(b) Evolution of overpressure (top) and rates of venting and expansion (bottom) extracted from LES

Figure 3: Distribution of pressure along the chamber and the mechanism driving the pressure rise: configuration B0BS.

Shown in Figure $3 \mathrm{~b}$ is the evolution of $\dot{V}_{\text {expa }}$ and $\dot{V}_{\text {vent }}$ with time during the explosion event. The overpressure is also shown to compare with the venting and expansion profiles. As it can be seen from Figure $3 \mathrm{~b}, \dot{V}_{\text {expa }}$ is only slightly greater than $\dot{V}_{\text {vent }}$ in the early stages of the explosion, so the chamber pressure slowly increases. The absence of B2 in configuration B0BS gives a relatively large separation distance between the two baffles. It leads to a slower rise in the combustion rate due to a long residence time so that the venting strength takes over the expansion at $t$ $\approx 3.15 \mathrm{~ms}$, and consequently, LES predicts a slower pressure rise close to B3. The intense flame-obstacle interaction after passing B3 leads to a sharp rise in the gas expansion. It also promotes the venting as the fast explosion flame pushes more un- 


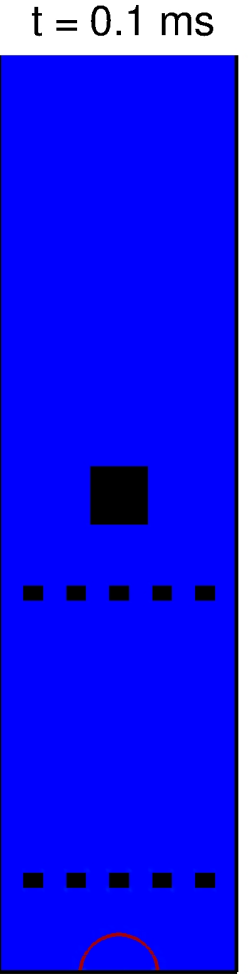

Vorticity $(1 / \mathrm{s})$ :
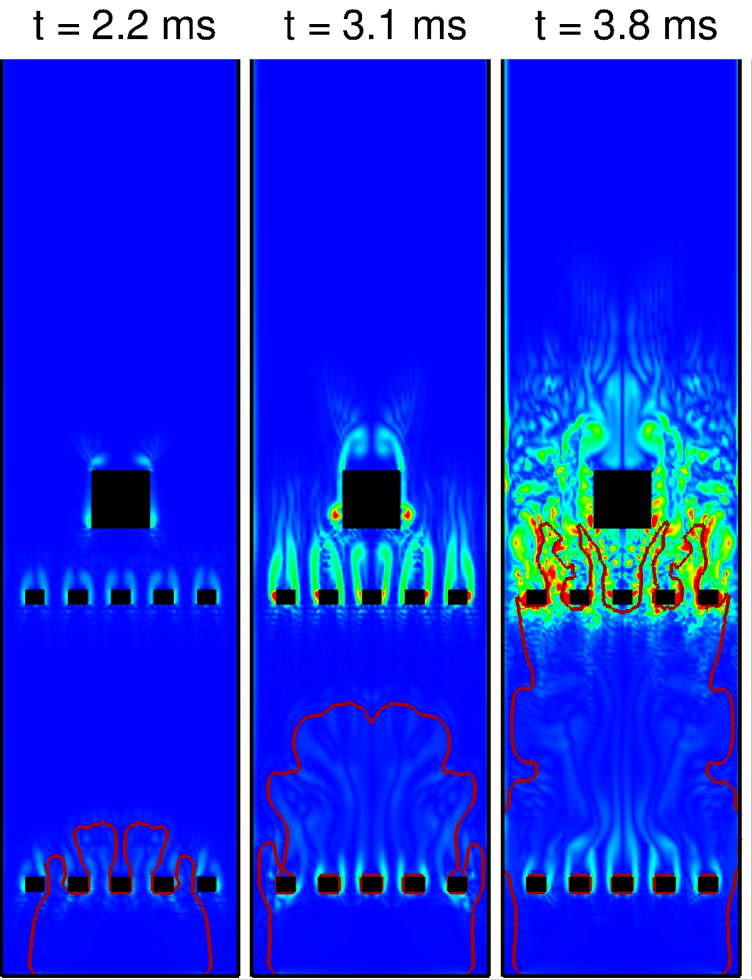

$\mathrm{t}=4.1 \mathrm{~ms}$

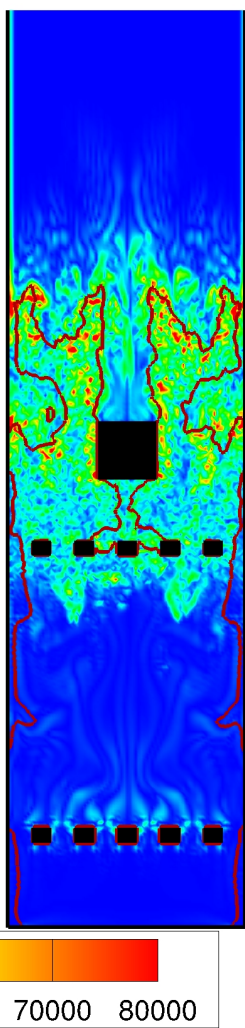

Figure 4: Numerical snapshots of the flame front represented by the iso-line of $\widetilde{c}=0.5$ with vorticity contours for configuration B0BS. Time instants correspond to $t=0.1,2.2,3.1,3.8$ and $4.1 \mathrm{~ms}$ (from left to right).

Figure 4 shows the flow dynamics and flame-flow interactions in the hydrogen explosion for the configuration B0BS extracted from LES. The recirculation zones 
and the turbulence level may be seen from the vorticity contour, and the flame front is defined using the iso-line of $\widetilde{c}$. As demonstrated earlier, the presence of obstacles may promote the combustion rate and significantly increase the explosion overpressure. The obstacles can induce vortices and turbulence of various length scales and strength. For instance, at $\mathrm{t}=2.2 \mathrm{~ms}$, weak recirculation regions behind the downstream obstacles can be observed even if the flame front is only interacting with the first grid. The turbulence intensity in the wake area of B3 and Sq.Ob. gradually becomes stronger with the rapid approach of the flame front. Intense flame-turbulence interactions make the flame surface wrinkle. As can be seen from $\mathrm{t}=3.8 \mathrm{~ms}$ in Figure 4, this not only speed up the flame but also creates more turbulence. Note that even in the downstream area of the last obstruction, the fast explosion is sustained by the continuous interactions between the propagating flame front and the generated turbulence in this region. As hydrogen explosions are usually rapid and highly unsteady, LES proves itself as a valuable tool in visualising the entire explosion process and in helping to understand its underlying physics.

\subsection{Overpressure and flame speed}

Figure 5 compares the maximum overpressure and its time of incidence between LES and the experiments for all the configurations (grouped in Table 1) studied in this paper. It is clear that the magnitude and timing of the peak pressure are strongly related to the configuration of the explosion chamber. Maximum pressure can increase by up to $400 \%$ by changing from configuration 000S to BBBL. In this case, the VBR changes from $1.15 \%$ (000S) to $6.44 \%$ (BBBL), and the peak overpressure increases from less than 200 mbar (000S) to $~ 1000$ mbar (BBBL). It is important to realise that a real explosion in hydrogen process plants is likely to occur in much larger scales. Thus, the generated maximum overpressure may be much higher in larger-scale scenarios. As can be seen from groups 1 and 2, the more congested the obstacles, the higher the overpressure peak it can reach. The position of the baffle plates also plays a vital role in determining the maximum pressure, e.g. groups 3 and 4. Generally, the peak pressure decreases when the grids are closer 

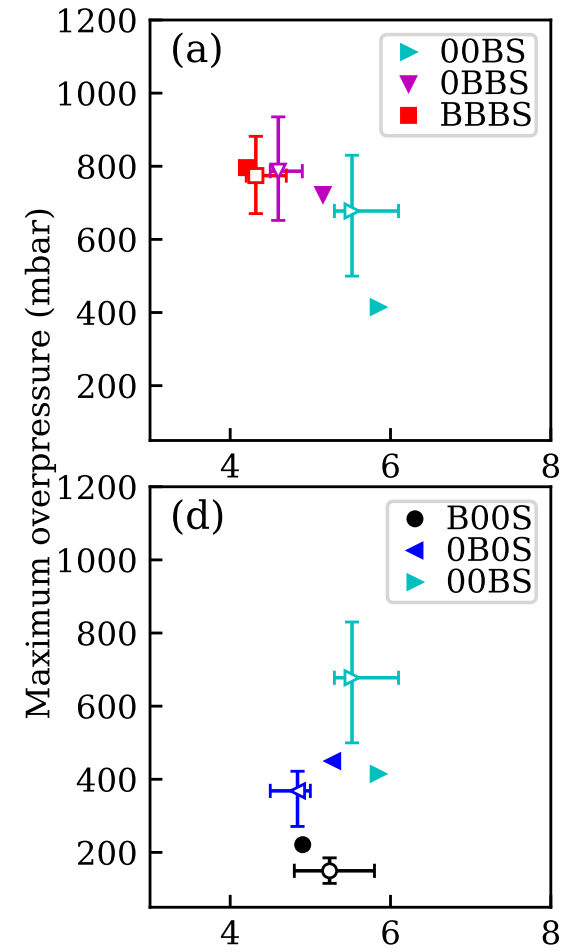
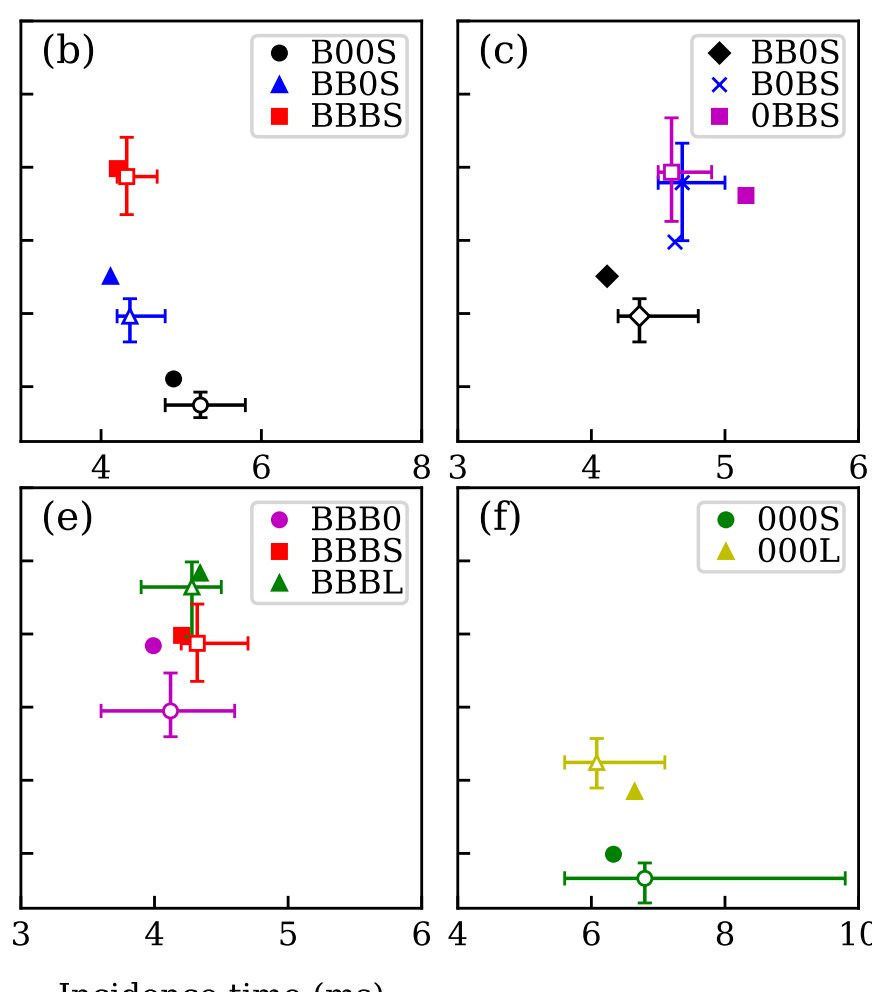

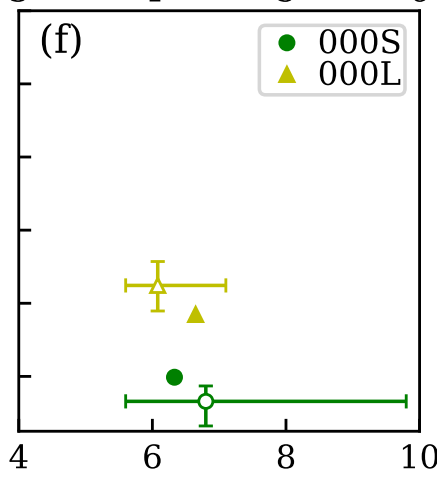

Figure 5: Comparison of the peak overpressure and its incidence time between the experiments and LES for all studied configurations: (a) group 1, (b) group 2, (c) group 3, (d) group 4, (e) group 5, and (f) group 6. Experimental mean values and variations (hollow symbols with error bars of time to peak and maximum overpressure).

Overall, LES gives satisfactory results compared with experiments regarding both the timing and magnitude of the maximum overpressure, and this implies that the applied DFSD model successfully accounts for the flame wrinkling in a range of flow configurations with various turbulence level. The numerical calculations give excellent predictions for cases such as 0BBS, BBBS and BBBL regarding the overpressure magnitude, while a small degree of over-estimation can be seen on the configuration BB0S, OBOS and BBB0. The most substantial discrepancy (absolute error) lies in the case 00BS where it is under-predicted by $\sim 250$ mbar. Calculated time taken to reach the peak pressure, $t_{p, L E S}$, is generally within or close to the range of the experimental measurement for all the test cases. Experimentally, the 
mean time taken to reach the peak overpressure varies approximately from 4 ms to 7 ms depending on the configuration. In general, one may expect that the higher the pressure peak, the shorter the time of occurrence. While it is true when the number of grids increases, i.e. groups 1 and 2, there are some exceptions where the relationship between the two is more complicated. For example, in group 3 (Figure 5c), the maximum pressure of BBOS is only half of OBBS, but its time of incidence is apparently shorter than the latter. As it can be seen, LES also shows a correct prediction behaviour consistent with the experimental results. Note that the variation in the timing and magnitude of the peak overpressure does exist in the experimental measurements, e.g. a comparatively wide range of $t_{p, \exp }$ for configuration 000S. Considering that LES results are from single realisation, factors including the initial turbulence level, numerical schemes and thermochemical properties of the fuel can affect the results to an extent. However, a complete parametric study to investigate all modelling options is beyond the scope of this paper. Thus, with the current modelling setup, the discrepancies shown in Figure 5 are thought to be acceptable. In general, all trends seen in experiments for crucial parameters such as peak pressure and time to peak are predicted reasonably well. Sections 4.2.1 and 4.2.2 will further discuss the influence of location, number and size of the obstruction, as well as the LES prediction.

\subsubsection{Effect of obstacle number and location}

This section focuses on the impact of the number and location of obstacles on the hydrogen-air explosion characteristics within the chamber. Note that the configurations in groups 1-4 (see Table 1) are equipped with a small central obstacle downstream of the baffles, representing various arrangements of turbulence-generating grids. Considering the conciseness of the paper and that the pressure trends are similar to an extent between configurations, results of overpressure and flame speed for groups 1 and 3 are not included in the main text but can be found in the supplementary material.

Figure 6 presents the pressure and flame speed evolutions for group 2 configura- 

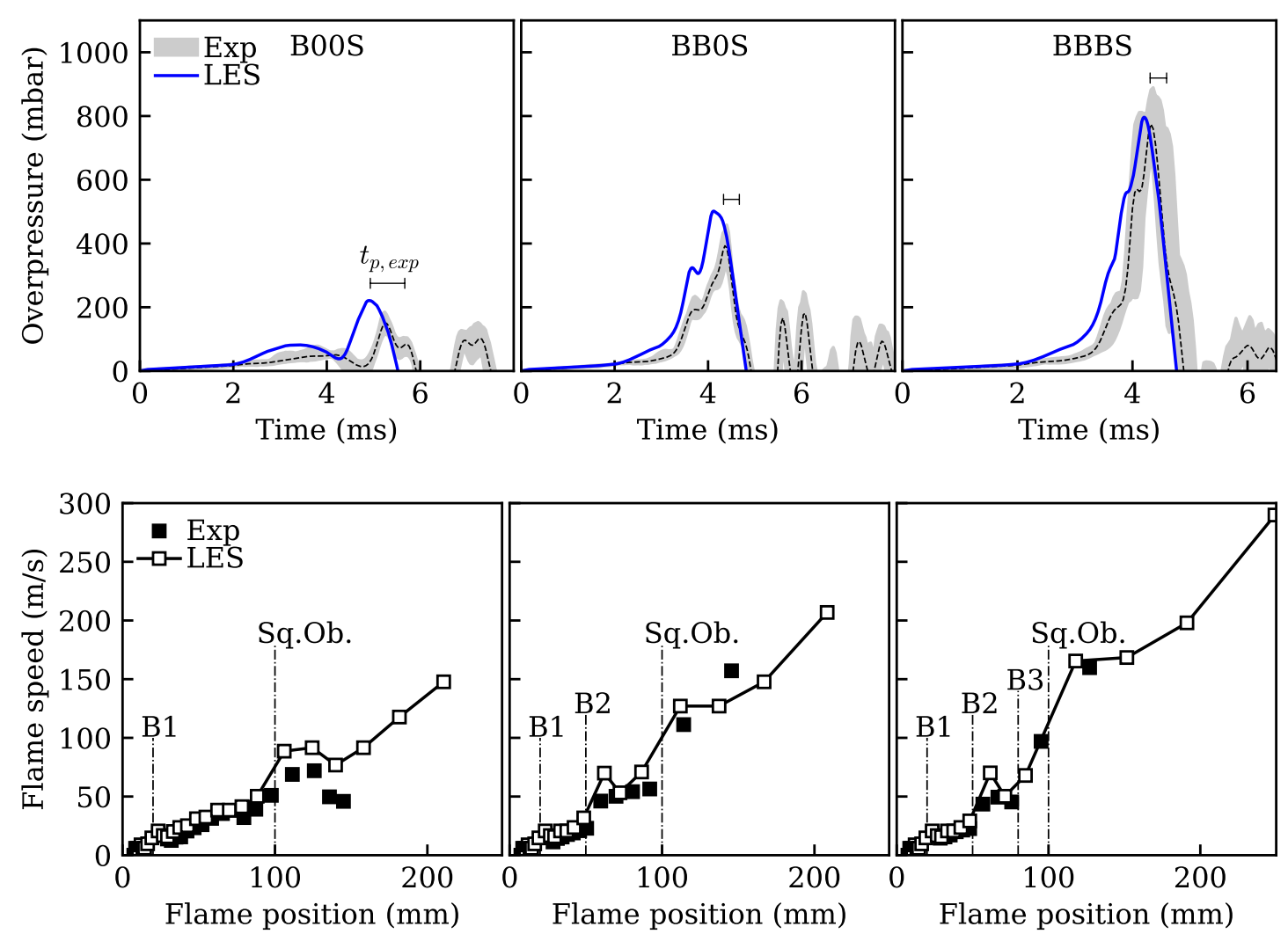

Figure 6: Comparison between LES and experiments for group 2. Top: Overpressure traces. Mean experimental signal (---). The variation in $t_{p, \exp }(\longmapsto)$. Bottom: Flame propagation speed. Locations of the baffle plates (B1, B2 and B3) and the central square obstacle (Sq.Ob.).

tions. It is worth noticing here that the mean overpressure for each case presented in this paper is averaged from 50 individual experimental realisations, and an illustration of the averaging process is provided in the supplementary material. As the pressure trends in each experiment are very similar [61], the averaging procedure is performed after shifting all the pressure traces in time to match the maximum overpressure of each one. The range of the overpressure magnitude and the time taken to reach the peak pressure $\left(t_{p, \text { exp }}\right)$ are also shown in the processed data (e.g. Figure 6) to facilitate the comparison with the numerical results. For flame speed profiles, the flame front position is numerically tracked by $\widetilde{c}=0.5$ furthest from the ignition end, while it is determined from the LIF-OH images experimentally.

Figure 6 shows that in group 2, the maximum pressure almost doubles when an additional grid is placed in the order starting closet to the ignition end. Comparison between the overpressure history and the corresponding flame speed indicates 
that considerable flame propagation speed commonly accompanies high overpressure. Therefore, additionally placed grids contribute to the higher flame speed in the region downstream of the central obstruction. Since the introduction of more obstructions (i.e. groups 1 and 2) in the path of the flame propagation increases the overall turbulence level generated within the chamber, the more intensive flameobstacle interactions give rise to the higher overpressure peak. Interestingly, both LES and experiments (Figure 5b) indicate that adding another grid to the case 0BBS (i.e. changing from $0 \mathrm{BBS}$ to $\mathrm{BBBS}$ ) does not significantly increase the overpressure peak, and this may reflect the weak effect of having the lower grid compared to the other two.

Another observation made from Figure 6 is that the hydrogen flame substantially accelerates after passing successive obstacles, resulting from the local blockage and the high reactivity of hydrogen. The velocity reaches $\sim 160 \mathrm{~m} / \mathrm{s}$ for the case BBBS just downstream of the square obstacle, and the flame can accelerate up to about $300 \mathrm{~m} / \mathrm{s}$ close to the exit as predicted by LES. The kinks in the speed profiles due to the temporary slowdown of the flame front right before it encounters an obstacle are also correctly reproduced numerically, indicating that the developed model successfully captures the essential dynamics of the flame propagation. Slight deviations in flame speeds from the experimental measurements can be noticed when the flame is downstream of the square obstacle in the blow-down area. It may be partially due to the limited time resolution of high-speed images considering the high turbulence and flame front speeds within the region.

Note that the exact mechanism of flame acceleration due to obstacles and confinement is complex. It may involve several types of flow instabilities such as the well-known Rayleigh-Taylor (RT) instability [62]. Furthermore, self-acceleration resulted from the intrinsic wrinkling of expanding laminar flame [62] may be present in the early development and quasi-laminar stage of the explosion. To the authors' knowledge, well-developed models are currently not available for these instabilities of the laminar flame [34]. However, for all the test cases considered here, the effect 

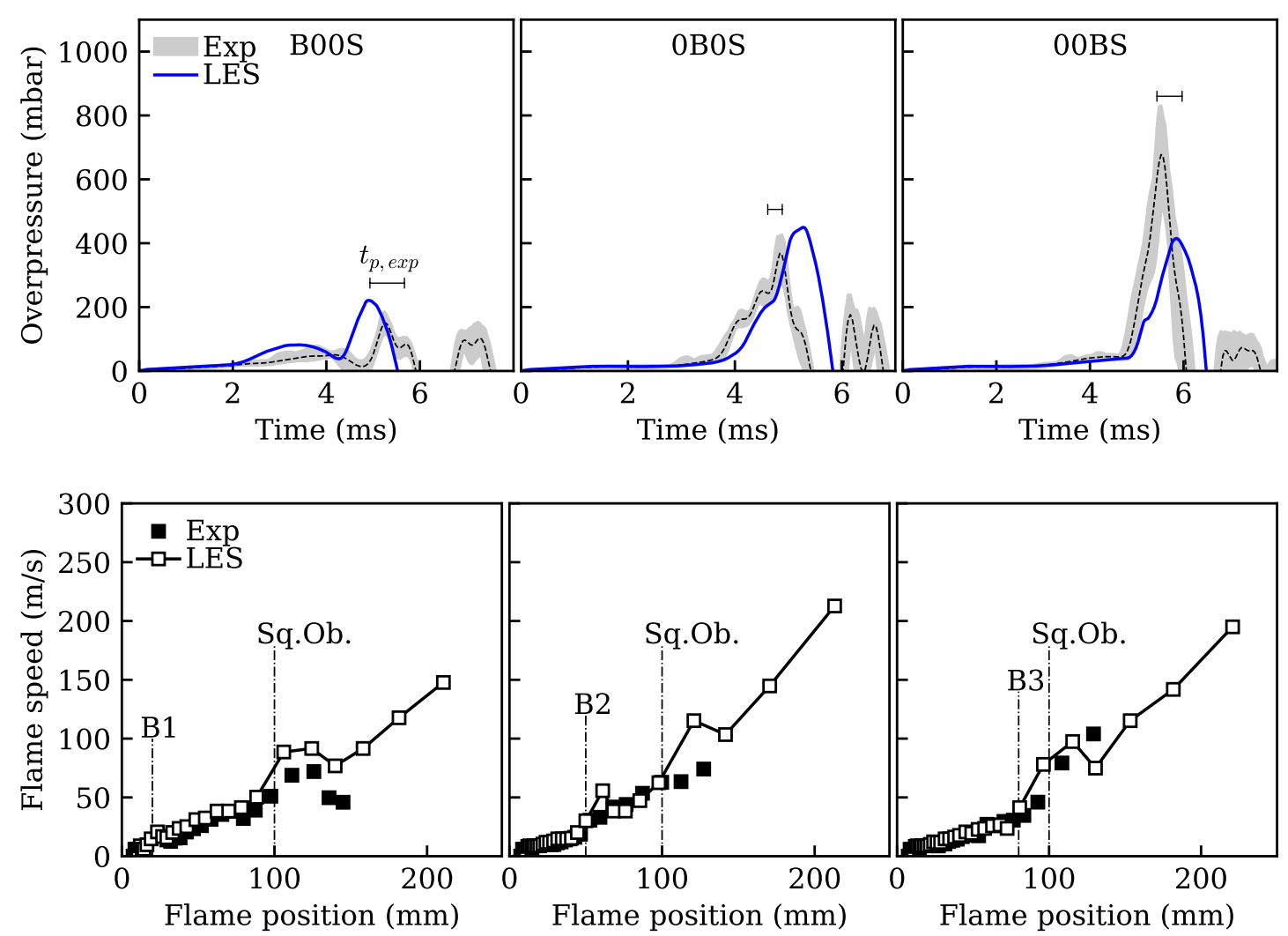

Figure 7: Comparison between LES and experiments for group 4. Top: overpressure traces. Mean experimental signal (---). The variation in $t_{p, e x p}(\longmapsto)$. Bottom: flame propagation speed. Locations of the baffle plates (B1, B2 and B3) and the central square obstacle (Sq.Ob.).

Recalling that Figure $5 \mathrm{c}$ demonstrates the effect of alternating the position of

of obstacle-generated turbulence is expected to be so dominant that the sub-grid contribution of the flame surface growth due to these instabilities may be neglected.

two baffle plates for group 3 configurations. Note that these cases have the same blockage ratio (i.e. VBR), and only the location of the grids varies. It clearly shows that higher overpressure is generated when the two baffles are closer to the central obstruction. Figure 7 provides a further illustration of the influence of the obstacle position using the group 4 configurations. It shows that the location of a single grid inside the chamber increases the pressure to different degrees. For the configuration B00S, due to the closeness of the lower baffle plate to the ignition source and the small thickness of the grid $(3 \mathrm{~mm})$, the flame front is only slightly stretched by B1 and then relaminarises as it passes. It is identified in Figure 7 by a noticeable bump in the pressure trace at $\sim 4 \mathrm{~ms}$ from both LES and experiments. It is also 
interesting to see that adding B1 to case 000S does not alter the peak overpressure very much (see Figure 5) even though the blockage ratio increases.

Based on Figures 6 and 7, LES has satisfactory performance in reproducing the overpressure trends, and even details such as small turning points in the pressure curves due to sudden changes in the burning rate are correctly captured. It reflects the main advantage of the DFSD model as the model coefficient accounting for the SGS flame wrinkling adjusts automatically in the simulation. It is particularly useful in the present test cases since the level of the flame front wrinkling can vary significantly for various configurations of obstacles.

As mentioned earlier, there appears to be an apparent underestimation $(\sim 250$ mbar less than the mean experimental value) in peak pressure for the configuration 00BS (Figure 5d) compared with the other cases. A closer look at its pressure trace in Figure 7 reveals that the early-stage overpressure matches well with the experiment despite the under-prediction when approaching the peak. Further investigation shows that the computed flame wrinkling factor for the case 00BS (see supplementary material) in the turbulent phase of the deflagration is of the similar magnitude as that of 0B0S in group 4. However, considering that the experimental peak pressure of the case 00BS is almost twice as large as that of 0B0S, the numerically under-predicted pressure peak may result from the underestimation of $\Xi_{\Delta}$ in the later stage of the explosion.

Figure 8 illustrates the effect of the single grid on the flame front structure just before contacting the square obstacle. For both cases of 000S and B00S, the flame front essentially travels to the square obstacle in a quasi-laminar manner. Although the greater vertical flow velocity of the latter indicates that the flame does propagate faster, it also promotes the venting from the top of the chamber. The efficient venting may also explain the lack of pressure generation by adding the first grid after the flame passes B1, resulting from the relatively long distance away from the next downstream obstruction. Accordingly, LES predicts a maximum overpressure of $\sim$ 200 mbar for both 000S and B00S despite that the experimental values are slightly 
lower (see Figure 5).

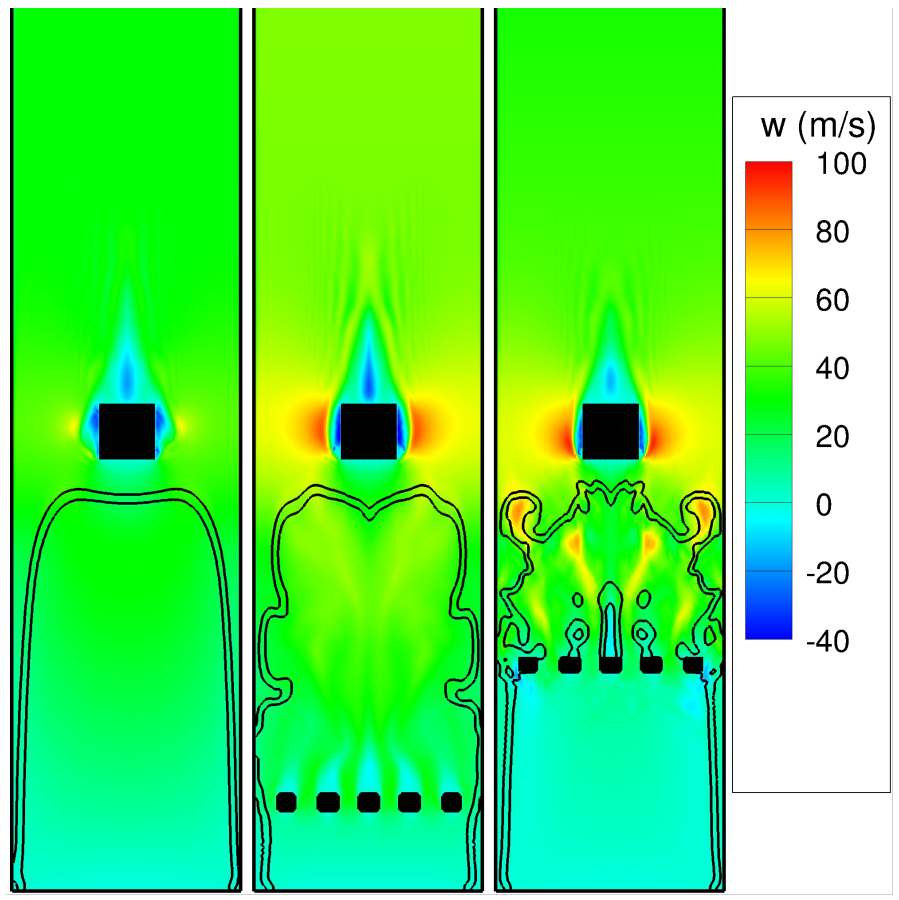

Figure 8: Effect of the single grid as illustrated by the flame front structure (iso-lines of $\widetilde{c}=0.2$ and $\widetilde{c}=0.8$ ) before reaching the square obstacle for configuration 000S, B00S and 0B0S (from left to right). Contours of negative axial velocity indicate recirculation regions in the flow.

A closer look at Figure 8 reveals that the flame structure substantially changes when moving the grid from the lower (B1) to the middle position (B2). Since the axial velocity $(\sim 40 \mathrm{~m} / \mathrm{s} . v s . \sim 10 \mathrm{~m} / \mathrm{s})$ at the baffle openings increases in time, the Reynolds number of the fresh gases based on the scale of the grid is higher. Furthermore, the formation of a few disconnected flame islands behind the baffle strips confirms the presence of stronger recirculation zone when flame-baffle interactions occur. It explains the more wrinkled and corrugated flame front for case 0B0S. The significant growth of the flame surface area before reaching the central obstacle also enhances their subsequent interactions, and the sub-grid wrinkling will be higher (see supplementary material). Consequently, the computed maximum overpressure of the case 0B0S is more than twice of that in the case B00S ( $~ 400$ mbar .vs. $\sim 200$ mbar), as also proved by the experiments. Therefore, it may be concluded that the distance between the grid and the ignition source serves as a controller and the generated overpressure is higher as the grid moves further downstream. 


\subsubsection{Influence of the obstacle size}

It is recalled from Table 1 that group 5 consists of configuration BBB0, BBBS and BBBL in which central obstacle starts from none to large, while group 6 constitutes configuration 000S and 000L where no baffles are present. As confirmed from the peak overpressure calculations and experiments (Figure 5e), increasing the size of the central obstruction enhances the peak pressure. On the other hand, when the central obstacle is the only obstruction in the chamber, the maximum overpressure is nearly doubled by using a large one according to Figure $5 \mathrm{f}$. In this case, the ABR almost doubled by changing from 000S to 000L, and the VBR of the latter (5\%) is about 4 times of the former $(\sim 1.2 \%)$. The computed pressure signals of group 5 are presented in Figure 9, while that of group 6 are included in the supplementary material.

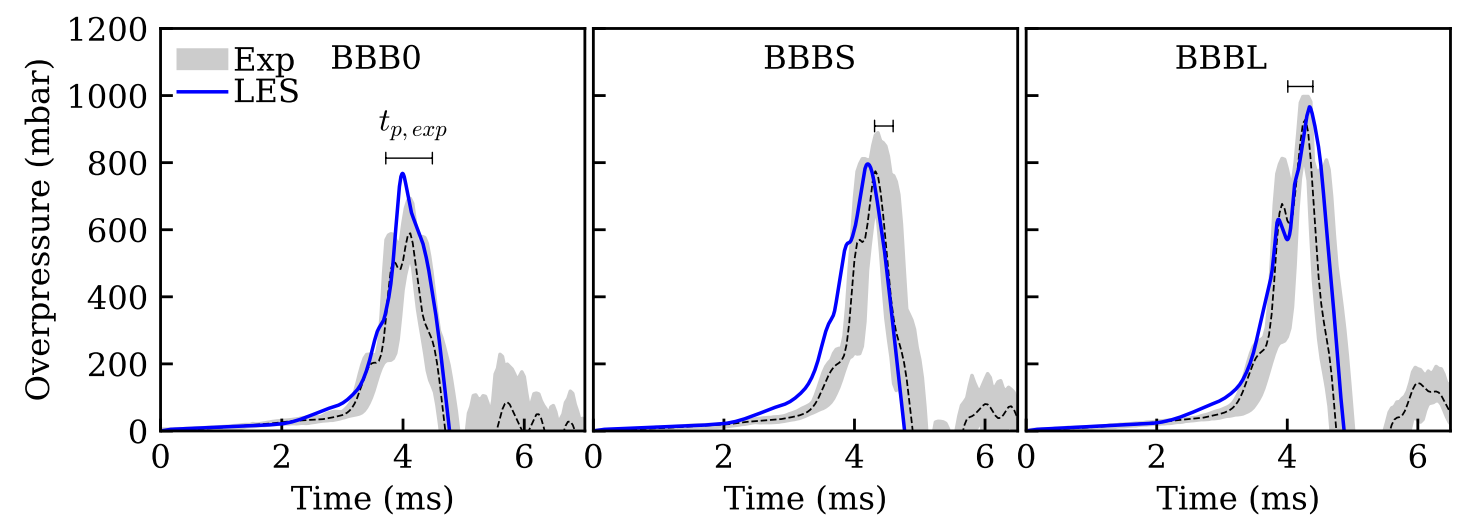

Figure 9: Comparison of overpressure signals between LES and experiments for group 5 cases. Mean experimental signal (--- ) . The variation in $t_{p, \exp }(\longmapsto)$.

Comparison between groups 5 and 6 (Figure 5e and f) reveals that with the presence of the three baffle plates, the maximum pressure is generally higher than the configurations with only the central obstruction. It shows the effectiveness of the baffles plates in creating turbulence and the contribution of continuous flameturbulence interaction to the internal pressure of the explosion chamber. The high turbulence intensity in the shear layer of the sharp-edged baffle strips leads to the substantially wrinkled flame front when reaching the central obstruction. Figure 5 also shows that the maximum pressure increases by $\sim 30 \%$ when adding a small 
obstacle to the case BBB0. A further rise of $\sim 20 \%$ is seen when the large one replaces it. It is worth mentioning that the overpressure generated by the configuration BBBL is the highest among all the cases and it can reach nearly 1 bar, and LES successfully captures the details on its pressure trace such as the sudden decrease in the overpressure at about $4 \mathrm{~ms}$ (Figure 9).

It is worth mentioning that both $\mathrm{ABR}$ and VBR are critical explosion-related parameters [63]. However, as has been demonstrated in section 4.2.1, the explosion overpressure is also influenced by the arrangement of obstacles in a multi-obstruction environment. Hence, LES is potentially a useful tool in complex obstructed process areas where simple correlations using parameters such as the blockage ratio are not sufficient.

Figure 10 shows the later stages of the explosion extracted from LES for configuration $\mathrm{BBB} 0, \mathrm{BBBS}$ and $\mathrm{BBBL}$ at various time instants. It can be seen that the deflagrating flame behaves differently based on the size of the obstacle at the central position. Without the central obstruction (i.e. configuration BBB0), the fingers-like flame fronts induced by B3 merge and it propagates towards the outlet. When an obstacle is placed, the flame tends to wrap around it and get further distorted. Note that the size of the obstacle plays a vital role in the flame behaviour. The passage between the side of the obstacle and the wall of the combustion chamber becomes narrower when the size is larger, causing the flame to be ejected from there at high speed. Furthermore, the large obstacle introduces a more extensive turbulent wake behind it where the flame is significantly wrinkled, and some unburned hydrogen-air mixture is trapped when passing it, as shown at $\mathrm{t}=3.9 \mathrm{~ms}$ from Figure 10 .

Figure 10d displays the flame location where the overpressure peaks ( $t \approx t_{p, L E S}$ ) for each configuration in the simulation. It can be seen that when the maximum pressure is reached, the flame is at a position closer to the outlet if the degree of obstruction is increased, and this may be due to the higher propagation speed of the flame front. For the case BBBL, the peak pressure is reached when the leading edge of the flame nearly at the exit of the chamber. Experimental findings [61] confirms 


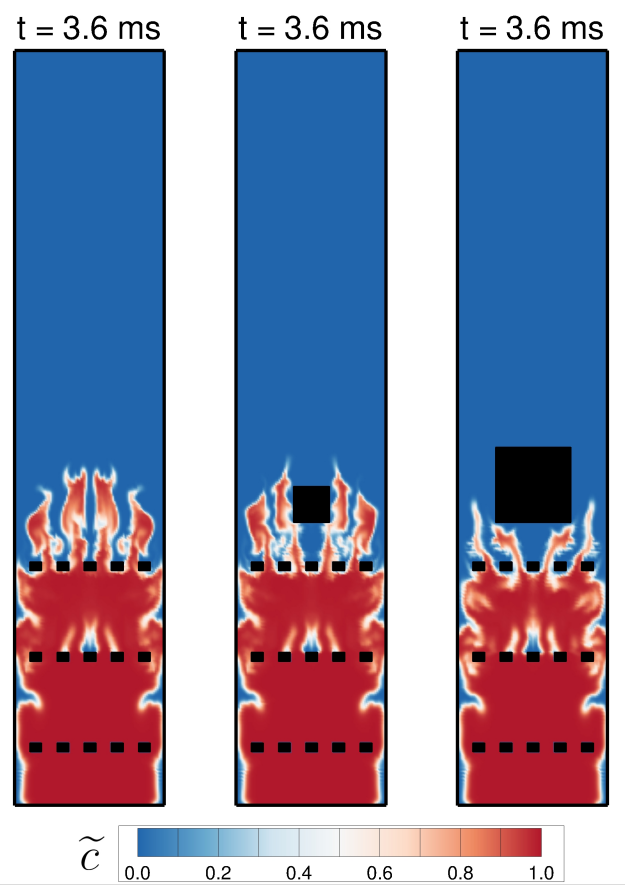

(a)

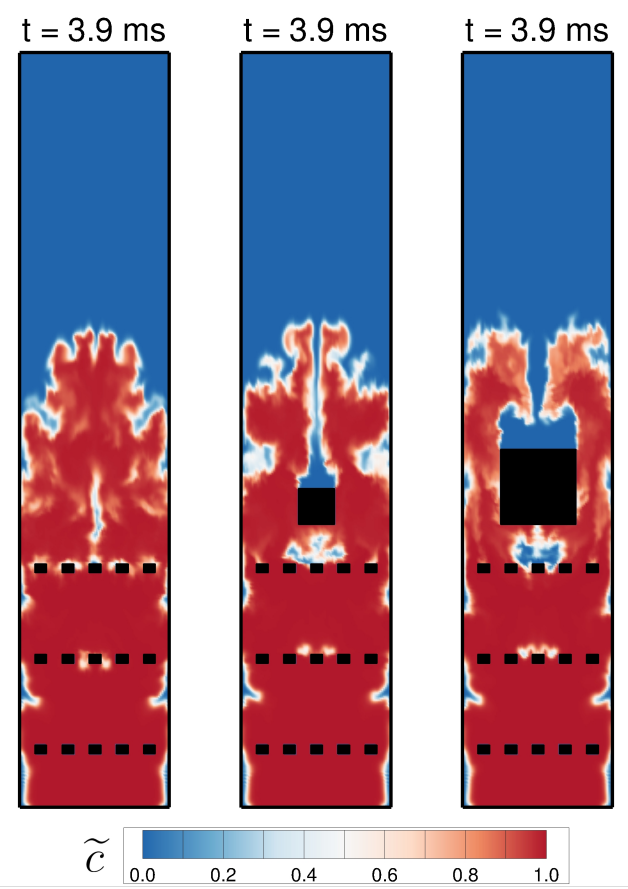

(c)

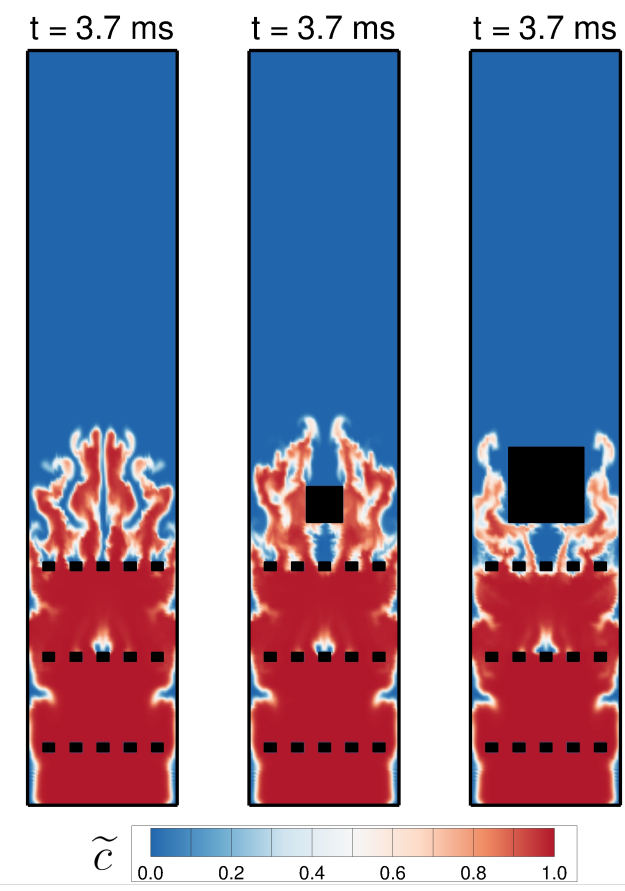

(b)

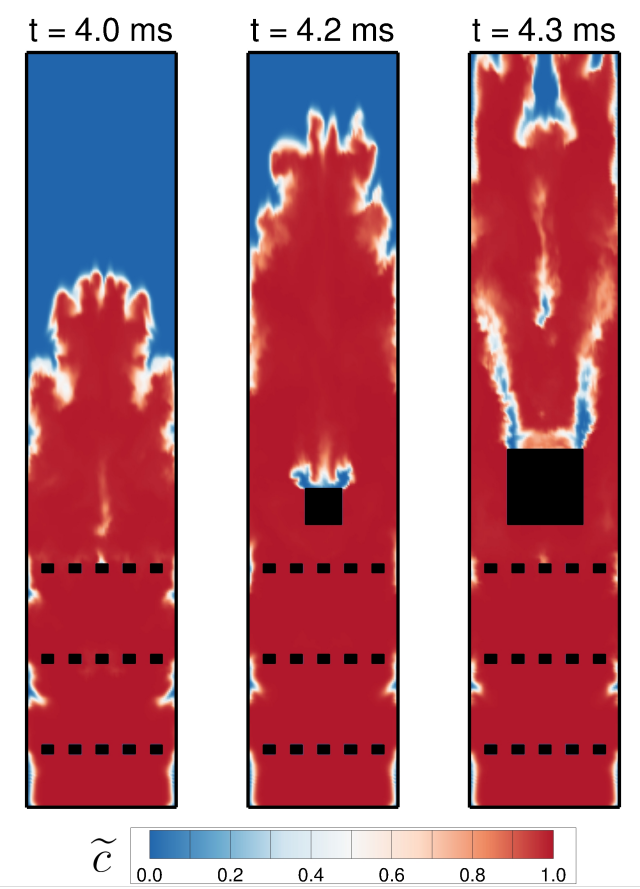

(d)

Figure 10: Flame snapshots (contoured by $\widetilde{c}$ ) of group 5 configurations (BBB0, BBBS and BBBL) showing the effect of the size of the central obstacle. (a) $t=3.6 \mathrm{~ms}$; (b) $t=3.7 \mathrm{~ms}$; (c) $t=3.9$ $\mathrm{ms} ;(\mathrm{d}) \mathrm{t} \approx \mathrm{t}_{p, L E S}$. 

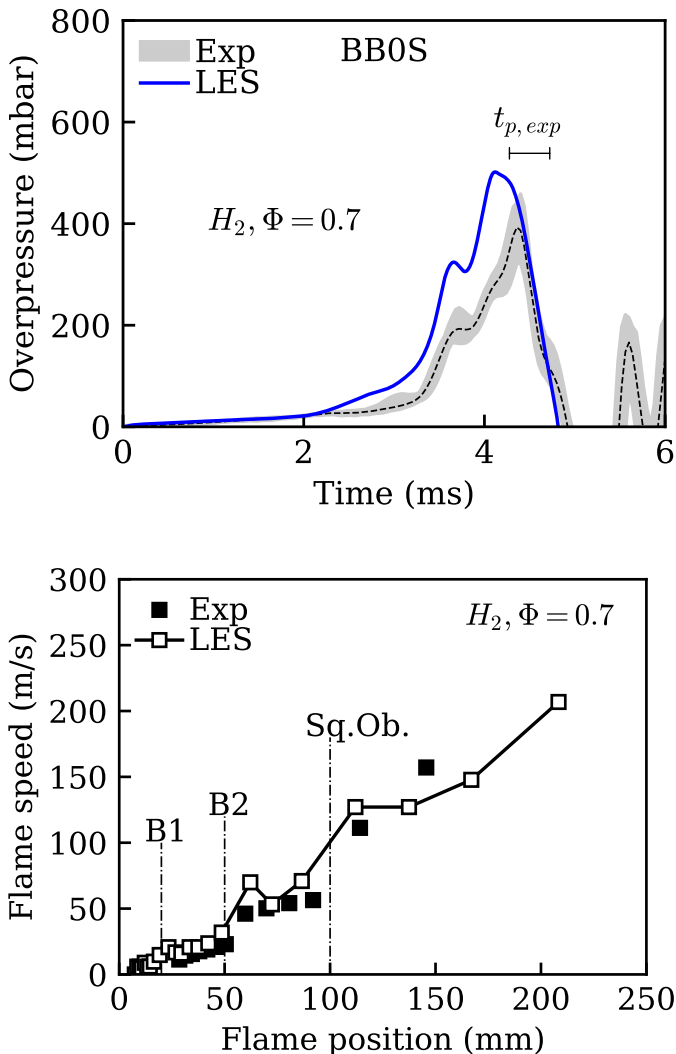
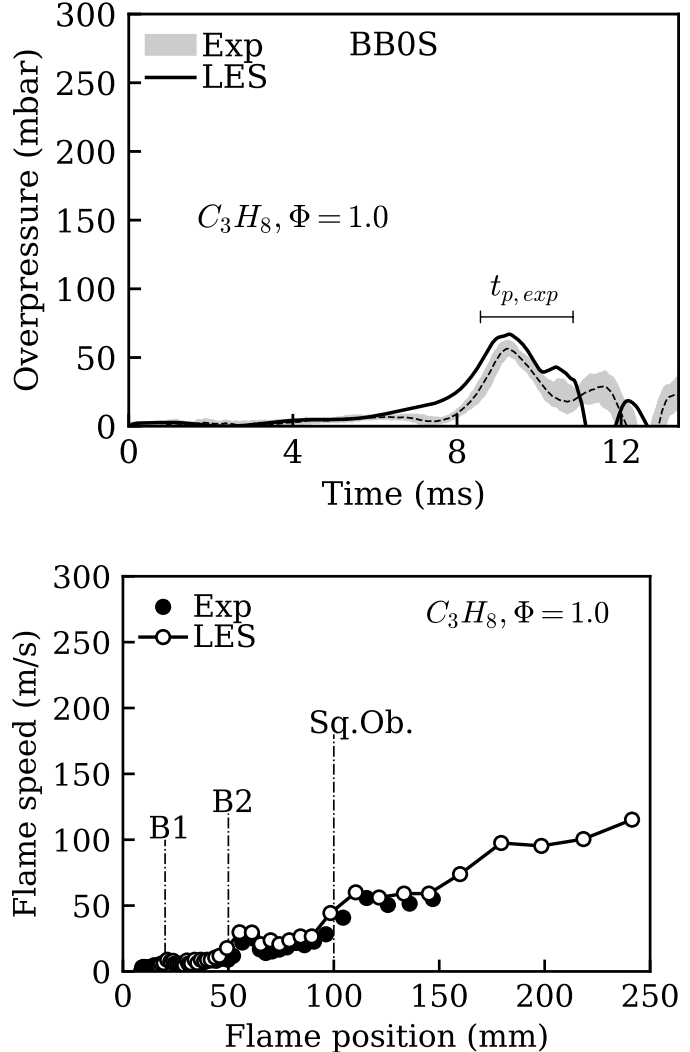

Figure 11: Comparison of overpressure signals and flame propagation speed between LES and experiments for configuration BB0S. Left: hydrogen, $\Phi=0.7$. Right: propane, $\Phi=1.0$. Mean experimental signal (-- - ). The variation in $t_{p, \exp }(\longmapsto)$. 
Figure 11 presents the overpressure and flame speed of configuration BB0S using the two fuel/air mixtures, showing the distinct explosion behaviours. It is apparent that even for stoichiometric propane/air mixture, the generated maximum overpressure is almost an order-of-magnitude lower ( $\sim 500$ mbar .vs. $\sim 60$ mbar $)$ than the lean hydrogen/air explosion. Also, the time taken to reach the peak is more than twice as slow as the hydrogen case, and the rate of pressure rise is considerably lower. As it can be seen from the speed-up locations in Figure 11, deflagrating hydrogen flame not only propagates faster but also responds more to the obstructions. The higher sensitivity to the obstacles for hydrogen leads to a magnified effect of flame-obstacle interactions compared with propane. While this section only includes one obstacle arrangement, the conclusions to the comparison between the two fuels drawn here are generally valid for other cases [61].

Note that the LES-DFSD approach excellently reproduces the experimental pressure trends, and it also captures the slightly different dynamics of the pressure traces for the same configuration between the two fuels. For example, it can be noticed from both LES and experiments in Figure 11 that there is a tiny pressure peak before the maxima at around $4 \mathrm{~ms}$ for hydrogen, while it is not present for propane. In contrast, a small peak can be found at about $10 \mathrm{~ms}$ for propane in the experiment, and it is also predicted numerically.

\section{Conclusions}

In this paper, large eddy simulations have been performed to investigate the vented hydrogen explosion in a small-scale obstructed chamber. A dynamic flame surface density model is applied to account for the progressive wrinkling of the flame front. The considered laboratory-scale explosion chamber has removable baffle plates and sharp-edge obstacles of different sizes, and a total of 11 flow configurations have been studied extensively. The highlight of the present work lies in the assessment of the recently developed LES-DFSD framework in modelling small-scale turbulent hydrogen explosions, as well as the insight into the mechanism driving the hydrogen 
explosion process.

Comparison between lean hydrogen-air $(\Phi=0.7)$ and stoichiometric propane-air $(\Phi=1.0)$ mixtures confirms that the magnitude of the peak pressure is an order higher for the configuration BB0S using hydrogen. Furthermore, the deflagrating flame of hydrogen is found to be more sensitive to the effect of obstacles compared with propane. Based on the numerical and the experimental studies discussed earlier, the following conclusions can be made on the influence of obstruction:

- The hydrogen flame is laminar at the beginning, but the degree of flame stretch and wrinkling increases as the flame front propagates past repeated baffles and obstacles. Thus, the explosion may involve different phases covering from quasilaminar to fully turbulent.

- Considerable flame propagation speed over successive obstructions generally links with strong overpressure.

- The peak overpressure grows with an increase in volume blockage ratio and with decreasing distance between successive obstructions. Maximum overpressure can be as low as $\sim 200$ mbar when only the small obstacle is present (i.e. configuration 000S). It may reach approximately 1 bar with the highest degree of obstruction (i.e. configuration BBBL).

- The overpressure is not only affected by the obstacle ABR and overall VBR, and the number of obstacles and their locations relative to the ignition source also influence the pressure build-up.

- Large separation between consecutive obstacles can lead to flame relaminarisation and flame deceleration, and the pressure may rise slowly or even decrease.

- The effect of the first grid on the peak pressure and flame velocity is small. Greater axial flow speeds (or the Reynolds number) and stronger recirculation zones at the baffle plate contribute to the higher level of flame-baffle interaction when the grid is moving downstream. 
- Size of the central obstacle affects both the peak overpressure and the location of the flame front when it is reached.

Comparison between the numerical and the experimental pressure histories and flame front speeds shows satisfactory agreement considering the variation and uncertainties in the experiments. It has been demonstrated that LES is capable of reproducing the critical features and dynamics of the hydrogen explosion process. The calculated overpressure corresponds well with the experimental data in both magnitude and trend over a range of obstacle arrangements. Encouragingly, LES also captures some details on the pressure-time curve such as sudden pressure rise and drop in the magnitude due to immediate changes in the burning rate. It is believed to be the capability of the LES-DFSD approach as the level of flame-turbulence interaction is appropriately accounted for at different stages of the explosion and also for configurations of various degree of obstructions. Satisfactory results obtained from LES encourages the application of the current models to study larger-scale hydrogen explosions with complex obstructions that are typically encountered in the actual hydrogen storage and processing areas.

\section{Nomenclature}

$\beta \quad$ Model coefficient in the DFSD model

$\Delta \quad$ LES combustion filter width

$\delta_{c} \quad$ Inner cut-off scale

$\delta_{L}^{0} \quad$ Laminar (thermal) flame thickness

$\Delta_{m} \quad$ Filter width of volume averaging

$\Delta_{x} \quad$ LES grid size

$\dot{\omega}_{c} \quad$ Reaction rate in terms of $c$

$\dot{V}_{\text {expa }} \quad$ Volumetric flow rate due to expansion

$\dot{V}_{\text {vent }} \quad$ Volumetric flow rate due to venting

Volume averaging operation

$\overrightarrow{\mathbf{n}} \quad$ Vector of normal direction

$\overrightarrow{\mathbf{u}} \quad$ Velocity vector 


\begin{tabular}{|c|c|c|}
\hline 648 & $\Omega$ & Volume of computational domain \\
\hline 649 & $\bar{\phi}$ & LES filtered quantity of $\phi$ (unweighted) \\
\hline 650 & $\Phi$ & Equivalence ratio \\
\hline 651 & $\rho$ & Density \\
\hline 652 & $\rho_{b}$ & Burned gas density \\
\hline 653 & $\rho_{u}$ & Unburned gas density \\
\hline 654 & $\Sigma$ & Flame surface density \\
\hline 655 & $\hat{\ldots}$ & Test-filtering operation \\
\hline 656 & $\widehat{\Delta}$ & Test-filter width \\
\hline 657 & $\widetilde{\phi}$ & LES filtered quantity of $\phi$ (mass weighted) \\
\hline 658 & $\Xi_{\Delta}$ & Sub-grid flame wrinkling factor \\
\hline 659 & $A$ & Area \\
\hline 660 & $A_{\text {exit }}$ & Area of chamber exit plane \\
\hline 661 & $c$ & Reaction progress variable \\
\hline 662 & $D$ & Molecular diffusion coefficient \\
\hline 663 & $S_{L}^{0}$ & Laminar burning velocity \\
\hline 664 & $t$ & Time \\
\hline 665 & $t_{p, \exp }$ & Time to reach peak pressure from experiments \\
\hline 666 & $t_{p, L E S}$ & Time to reach peak pressure from LES \\
\hline 667 & $u_{i}$ & Velocity component \\
\hline 668 & $V$ & Volume \\
\hline 669 & $V_{b}$ & Volume occupied by burned gas \\
\hline 670 & $x_{i}$ & Direction in Cartesian coordinate \\
\hline 671 & ABR & Area blockage ratio \\
\hline 672 & B1 & Baffle plate no. 1 \\
\hline 673 & $\mathrm{~B} 2$ & Baffle plate no. 2 \\
\hline 674 & B3 & Baffle plate no. 3 \\
\hline 675 & CFD & Computational fluid dynamics \\
\hline 676 & DFSD & Dynamic flame surface density \\
\hline
\end{tabular}




$\begin{array}{lll}677 & \text { FSD } & \text { Flame surface density } \\ 678 & \text { L } & \text { Large central obstacle } \\ 679 & \text { LES } & \text { Large eddy simulation } \\ 680 & \text { LIF-OH } & \text { Laser-induced fluorescence of OH } \\ 681 & \text { LPG } & \text { Liquid petroleum gas } \\ 682 & \text { RANS } & \text { Reynolds-averaged Navier-Stokes } \\ 683 & \text { S, Sq.Obs } & \text { Small central obstacle } \\ 684 & \text { SGS } & \text { Sub-grid scale } \\ { }_{685} & \text { VBR } & \text { Volume blockage ratio }\end{array}$

\section{Acknowledgement}

The authors would like to thank Dr Bo Liu from the University of Sheffield for his valuable suggestions on the modelling conducted in this paper. The original experimental data provided by Professor Assaad Masri from the University of Sydney are gratefully appreciated.

\section{Reference}

[1] G. Marbán, T. Valdés-Solís, Towards the hydrogen economy?, International Journal of Hydrogen Energy 32 (2007) 1625-1637.

[2] A. Veziroglu, R. MacArio, Fuel cell vehicles: State of the art with economic and environmental concerns, International Journal of Hydrogen Energy 36 (2011) $25-43$.

[3] T. E. Lipman, M. Elke, J. Lidicker, Hydrogen fuel cell electric vehicle performance and user-response assessment: Results of an extended driver study, International Journal of Hydrogen Energy 43 (2018) 12442-12454.

[4] C. M. White, R. R. Steeper, A. E. Lutz, The hydrogen-fueled internal combustion engine: a technical review, International Journal of Hydrogen Energy 31 (2006) 1292-1305.

[5] P. E. Dodds, I. Staffell, A. D. Hawkes, F. Li, P. Grünewald, W. McDowall, P. Ekins, Hydrogen and fuel cell technologies for heating: A review, International Journal of Hydrogen Energy 40 (2015) 2065-2083.

[6] Y. Lee, J. Kim, J. Kim, E. J. Kim, Y. G. Kim, I. Moon, Development of a webbased 3D virtual reality program for hydrogen station, International Journal of Hydrogen Energy 35 (2010) 2112-2118. 
[7] E. Kim, J. Park, J. H. Cho, I. Moon, Simulation of hydrogen leak and explosion for the safety design of hydrogen fueling station in Korea, International Journal of Hydrogen Energy 38 (2013) 1737-1743.

[8] B. Angers, A. Hourri, P. Benard, E. Demaël, S. Ruban, S. Jallais, Modeling of hydrogen explosion on a pressure swing adsorption facility, International Journal of Hydrogen Energy 39 (2014) 6210-6221.

[9] S. Kikukawa, H. Mitsuhashi, A. Miyake, Risk assessment for liquid hydrogen fueling stations, International Journal of Hydrogen Energy 34 (2009) 11351141.

[10] Y. F. Khalil, Science-based framework for ensuring safe use of hydrogen as an energy carrier and an emission-free transportation fuel, Process Safety and Environmental Protection 117 (2018) 326-340.

[11] A. G. Venetsanos, D. Baraldi, P. Adams, P. S. Heggem, H. Wilkening, CFD modelling of hydrogen release, dispersion and combustion for automotive scenarios, Journal of Loss Prevention in the Process Industries 21 (2008) 162-184.

[12] J. Yanez, M. Kuznetsov, A. Souto-Iglesias, An analysis of the hydrogen explosion in the Fukushima-Daiichi accident, International Journal of Hydrogen Energy 40 (2015) 8261-8280.

[13] O. R. Hansen, M. T. Kjellander, J. A. Pappas, Explosion loading on equipment from CFD simulations, Journal of Loss Prevention in the Process Industries 44 (2016) 601-613.

[14] O. J. Ugarte, V. Akkerman, A. S. Rangwala, A computational platform for gas explosion venting, Process Safety and Environmental Protection 99 (2016) $167-174$.

[15] C. R. Bauwens, J. Chaffee, S. B. Dorofeev, Vented explosion overpressures from combustion of hydrogen and hydrocarbon mixtures, International Journal of Hydrogen Energy 36 (2011) 2329-2336.

[16] J. Guo, Q. Li, D. Chen, K. Hu, K. Shao, C. Guo, C. Wang, Effect of burst pressure on vented hydrogen-air explosion in a cylindrical vessel, International Journal of Hydrogen Energy 40 (2015) 6478-6486.

[17] J. Guo, X. Sun, S. Rui, Y. Cao, K. Hu, C. Wang, Effect of ignition position on vented hydrogen-air explosions, International Journal of Hydrogen Energy 40 (2015) 15780-15788.

[18] X. Rocourt, I. Sochet, B. Pellegrinelli, Small-scale flame acceleration and application of medium and large-scale flame speed correlations, International Journal of Hydrogen Energy 42 (2017) 1327-1336.

[19] X. Lv, L. Zheng, Y. Zhang, M. Yu, Y. Su, Combined effects of obstacle position and equivalence ratio on overpressure of premixed hydrogenair explosion, International Journal of Hydrogen Energy 41 (2016) 17740-17749. 
[20] N. R. Popat, C. A. Catlin, B. J. Arntzen, R. P. Lindstedt, B. H. Hjertager, T. Solberg, O. Saeter, A. C. Van Den Berg, Investigations to improve and assess the accuracy of computational fluid dynamic based explosion models, Journal of Hazardous Materials 45 (1996) 1-25.

[21] B. J. Arntzen, Modelling of turbulence and combustion for simulation of gas explosions in complex geometries, Ph.D. thesis, Norwegian University of Science and Technology, 1998. URL: http://ntnu . diva-portal .org/smash/record.jsf?pid=diva2:122240\{\%\}5Cnhttp: //ntnu.diva-portal.org/smash/get/diva2:122240/FULLTEXT01. doi:10.1016/j.jlp.2005.06.012.

[22] L. Sun, H. Yan, S. Liu, Y. Bai, Load characteristics in process modules of offshore platforms under jet fire: The numerical study, Journal of Loss Prevention in the Process Industries 47 (2017) 29-40.

[23] K. B. Mishra, The influence of Volume Blockage Ratio on IOCL Jaipur explosion, Journal of Loss Prevention in the Process Industries (2018) - -

[24] P. Middha, O. R. Hansen, I. E. Storvik, Validation of CFD-model for hydrogen dispersion, Journal of Loss Prevention in the Process Industries 22 (2009) 1034-1038.

[25] K. Matsuura, H. Kanayama, H. Tsukikawa, M. Inoue, Numerical simulation of leaking hydrogen dispersion behavior in a partially open space, International Journal of Hydrogen Energy 33 (2008) 240-247.

[26] T. Beard, M. Bragin, W. Malalasekera, S. S. Ibrahim, Numerical Simulation of Hydrogen Discharge in a Partially Enclosed Space, Energy Procedia 66 (2015) $153-156$.

[27] M. A. Abdel-Raheem, S. S. Ibrahim, W. Malalasekera, A. R. Masri, Large Eddy simulation of hydrogen-air premixed flames in a small scale combustion chamber, International Journal of Hydrogen Energy 40 (2015) 3098-3109.

[28] K. K. J. Ranga Dinesh, X. Jiang, J. A. Van Oijen, Numerical simulation of hydrogen impinging jet flame using flamelet generated manifold reduction, International Journal of Hydrogen Energy 37 (2012) 4502-4515.

[29] E. Vyazmina, S. Jallais, Validation and recommendations for FLACS CFD and engineering approaches to model hydrogen vented explosions: Effects of concentration, obstruction vent area and ignition position, International Journal of Hydrogen Energy 41 (2016) 15101-15109.

[30] A. Marangon, M. Schiavetti, M. Carcassi, P. Pittiglio, P. Bragatto, A. Castellano, Turbulent hydrogen deflagration induced by ostacles in real confined environment, International Journal of Hydrogen Energy 34 (2009) 4669-4674.

[31] J. Li, F. Hernandez, H. Hao, Q. Fang, H. Xiang, Z. Li, X. Zhang, L. Chen, Vented Methane-air Explosion Overpressure Calculation-A simplified approach based on CFD, Process Safety and Environmental Protection 109 (2017) 489508. 
[32] D. Baraldi, D. Melideo, A. Kotchourko, K. Ren, J. Yanez, O. Jedicke, S. G. Giannissi, I. C. Tolias, A. G. Venetsanos, J. Keenan, D. Makarov, V. Molkov, S. Slater, F. Verbecke, A. Duclos, Development of a model evaluation protocol for CFD analysis of hydrogen safety issues the SUSANA project, International Journal of Hydrogen Energy 42 (2017) 7633-7643.

[33] D. Baraldi, A. Kotchourko, A. Lelyakin, J. Yanez, A. Gavrikov, A. Efimenko, F. Verbecke, D. Makarov, V. Molkov, A. Teodorczyk, An inter-comparison exercise on CFD model capabilities to simulate hydrogen deflagrations with pressure relief vents, International Journal of Hydrogen Energy 35 (2010) 12381-12390.

[34] R. S. Cant, W. N. Dawes, A. M. Savill, Advanced CFD and Modeling of Accidental Explosions, Annual Review of Fluid Mechanics 36 (2004) 97-119.

[35] H. Hisken, G. A. Enstad, P. Middha, K. Van Wingerden, Investigation of concentration effects on the flame acceleration in vented channels, Journal of Loss Prevention in the Process Industries 36 (2014) 447-459.

[36] J. Williamson, J. Mcgill, A. Trouve, Large Eddy Simulation Modeling of Turbulent Deflagrations, in: Fire Safety Science - Proceedings of the Eighth International Symposium, 2005, pp. 1375-1386. URL: http: //iafss.org/publications/fss/8/1375/view/fss\{_\}8-1375.pdf. doi:10. 3801/IAFSS.FSS. 8-1375.

[37] V. Molkov, D. Makarov, J. Puttock, The nature and large eddy simulation of coherent deflagrations in a vented enclosure-atmosphere system, Journal of Loss Prevention in the Process Industries 19 (2006) 121-129.

[38] D. Makarov, V. Molkov, Y. Gostintsev, Comparison Between Rng and Fractal Combustion Models for Les of Unconfined Explosions, Combustion Science and Technology 179 (2007) 401-416.

[39] V. Molkov, F. Verbecke, D. Makarov, LES of Hydrogen-Air Deflagrations in a 78.5-m Tunnel, Combustion Science and Technology 180 (2008) 796-808.

[40] S. R. Gubba, S. S. Ibrahim, W. Malalasekera, A. R. Masri, LES Modeling of Premixed Deflagrating Flames in a Small-Scale Vented Explosion Chamber with a Series of Solid Obstructions, Combustion Science and Technology 180 (2008) 1936-1955.

[41] V. Di Sarli, A. Di Benedetto, G. Russo, Using Large Eddy Simulation for understanding vented gas explosions in the presence of obstacles, Journal of Hazardous Materials 169 (2009) 435-442.

[42] R. Li, W. Malalasekera, S. Ibrahim, B. Liu, On the mechanism of pressure rise in vented explosions : A numerical study, Process Safety and Environmental Protection 117 (2018) 551-564.

[43] S. Patel, S. Jarvis, S. Ibrahim, G. Hargrave, An experimental and numerical investigation of premixed flame deflagration in a semiconfined explosion chamber, Proceedings of the Combustion Institute 29 (2002) 1849-1854. 
[44] H. Pitsch, Large-Eddy Simulation of Turbulent Combustion, Annual Review of Fluid Mechanics 38 (2005) 453-482.

[45] M. Boger, D. Veynante, Large eddy simulations of a turbulent premixed vshape flame, in: C. Dopazo (Ed.), Eighth European Turbulence Conference, Eighth European Turbulence Conference, Barcelona, Spain, 2000, pp. 448-452. doi:10.1016/0142-727X (90)90030-F.

[46] M. P. Kirkpatrick, S. W. Armfield, A. R. Masri, S. S. Ibrahim, Large Eddy Simulation of a Propagating Turbulent Premixed Flame, Flow, Turbulence and Combustion 70 (2003) 1-19.

[47] M. Boger, D. Veynante, H. Boughanem, A. Trouvé, Direct numerical simulation analysis of flame surface density concept for large eddy simulation of turbulent premixed combustion, Symposium (International) on Combustion 27 (1998) $917-925$.

[48] T. Ma, O. T. Stein, N. Chakraborty, A. M. Kempf, A posteriori testing of algebraic flame surface density models for LES, Combustion Theory and Modelling 17 (2013) 431-482.

[49] E. R. Hawkes, R. S. Cant, A flame surface density approach to large-eddy simulation of premixed turbulent combustion, Proceedings of the Combustion Institute 28 (2000) 51-58.

[50] G. Wang, M. Boileau, D. Veynante, K. Truffin, Large eddy simulation of a growing turbulent premixed flame kernel using a dynamic flame surface density model, Combustion and Flame 159 (2012) 2742-2754.

[51] A. Alharbi, A. R. Masri, S. S. Ibrahim, Turbulent premixed flames of CNG, LPG, and H2 propagating past repeated obstacles, Experimental Thermal and Fluid Science 56 (2014) 2-8.

[52] D. Veynante, V. Moureau, Analysis of dynamic models for large eddy simulations of turbulent premixed combustion, Combustion and Flame 162 (2015) 4622-4642.

[53] G. Wang, M. Boileau, D. Veynante, Implementation of a dynamic thickened flame model for large eddy simulations of turbulent premixed combustion, Combustion and Flame 158 (2011) 2199-2213.

[54] K. T. Aung, M. I. Hassan, G. M. Faeth, Flame stretch interactions of laminar premixed hydrogen/air flames at normal temperature and pressure, Combustion and Flame 109 (1997) 1-24.

[55] O. Vermorel, P. Quillatre, T. Poinsot, LES of explosions in venting chamber: A test case for premixed turbulent combustion models, Combustion and Flame 183 (2017) 207-223.

[56] M. P. Kirkpatrick, A large eddy simulation code for industrial and environmental flows, Ph.D. thesis, The University of Sydney, 2002. 
[57] H. Werner, H. Wengle, Large-Eddy Simulation of Turbulent Flow Over and Around a Cube in a Plate Channel, in: F. Durst, R. Friedrich, B. E. Launder, F. W. Schmidt, U. Schumann, J. H. Whitelaw (Eds.), Turbulent Shear Flows 8, Springer Berlin Heidelberg, Berlin, Heidelberg, 1993, pp. 155-168.

[58] M. Germano, U. Piomelli, P. Moin, W. H. Cabot, A dynamic subgrid-scale eddy viscosity model, Physics of Fluids A: Fluid Dynamics 3 (1991) 1760-1765.

[59] B. P. Leonard, A stable and accurate convective modelling procedure based on quadratic upstream interpolation, Computer methods in applied mechanics and engineering 19 (1979) 59-98.

[60] S. B. Pope, Ten questions concerning the large-eddy simulation of turbulent flows, New Journal of Physics 6 (2004).

[61] A. Alharbi, Turbulent Premixed Flames Propagating Past Repeated Obstacles, Ph.D. thesis, University of Sydney, 2013. URL: http://hdl .handle.net/ $2123 / 9765$.

[62] S. B. Dorofeev, Flame acceleration and explosion safety applications, Proceedings of the Combustion Institute 33 (2011) 2161-2175.

[63] D. Bjerketvedt, J. R. Bakke, K. van Wingerden, Gas explosion handbook, Journal of Hazardous Materials 52 (1997) 1-150. 